\title{
Metaheuristics for the Generalized Quadratic Assignment Problem
}

\author{
Roseline Mostafa \\ West Virginia University, rm1027@mix.wvu.edu
}

Follow this and additional works at: https://researchrepository.wvu.edu/etd

Part of the Industrial Engineering Commons, and the Operational Research Commons

\section{Recommended Citation}

Mostafa, Roseline, "Metaheuristics for the Generalized Quadratic Assignment Problem" (2020). Graduate Theses, Dissertations, and Problem Reports. 7717.

https://researchrepository.wvu.edu/etd/7717

This Thesis is protected by copyright and/or related rights. It has been brought to you by the The Research Repository @ WVU with permission from the rights-holder(s). You are free to use this Thesis in any way that is permitted by the copyright and related rights legislation that applies to your use. For other uses you must obtain permission from the rights-holder(s) directly, unless additional rights are indicated by a Creative Commons license in the record and/ or on the work itself. This Thesis has been accepted for inclusion in WVU Graduate Theses, Dissertations, and Problem Reports collection by an authorized administrator of The Research Repository @ WVU. For more information, please contact researchrepository@mail.wvu.edu. 


\title{
Metaheuristics for the Generalized Quadratic Assignment Problem
}

\author{
Roseline Mostafa
}

\author{
Thesis submitted to the \\ College of Engineering and Mineral Resources \\ at West Virginia University \\ in partial fulfillment of the requirements \\ for the degree of \\ Master of Science \\ in \\ Industrial Engineering
}

\author{
Alan McKendall, Ph.D., Chair \\ Kenneth R. Currie, Ph.D., P.E. \\ Zhichao Liu, Ph.D. \\ Department of Industrial and Management Systems Engineering
}

\author{
Morgantown, West Virginia
}

July 2020

Keywords: Generalized Quadratic Assignment Problem, Metaheuristics, Tabu Search, Simulated Annealing

Copyright 2020 Roseline Mostafa 


\section{ABSTRACT \\ Metaheuristics for the Generalized Quadratic Assignment Problem}

\section{Roseline Mostafa}

The generalized quadratic assignment problem (GQAP) is the task of assigning a set of facilities to a set of locations such that the sum of the assignment and transportation costs is minimized. The facilities may have different space requirements, and the locations may have varying space capacities. Also, multiple facilities may be assigned to each location such that space capacity is not exceeded. In this research, an application of the GQAP is presented for assigning a set of machines to a set of locations on the plant floor. Two meta-heuristics are proposed for solving the GQAP: tabu search (TS) and simulated annealing (SA). In addition, two types of neighborhood structures are considered for each meta-heuristic. A set of 21 test problems, available in the literature, is used to evaluate the performances of the meta-heuristics using one or two neighborhood structures. Computational experiments show that the proposed SA heuristics performed better than the proposed TS heuristics. The SA heuristics obtained results better than those presented in the literature for three of the test problems. On the other hand, the TS heuristics did not perform well for the problems with high space capacity utilization. 


\section{TABLE OF CONTENTS}

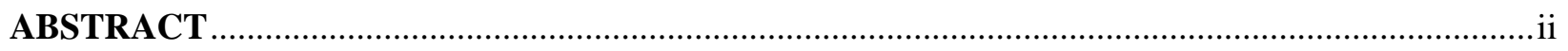

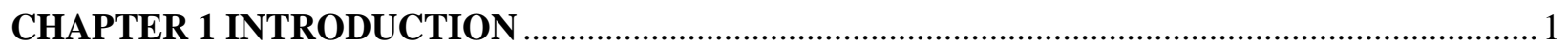

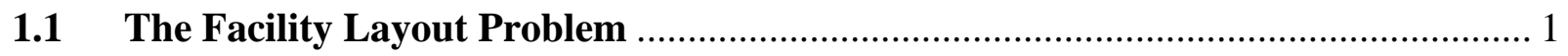

1.2 The Importance of the Facility Layout Problem ................................................... 2

1.3 The Generalized Quadratic Assignment Problem ............................................... 3

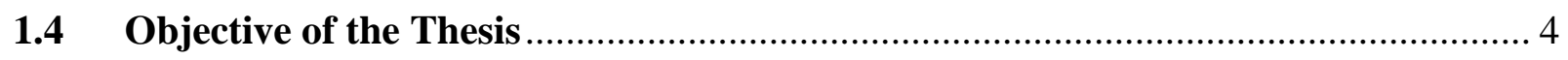

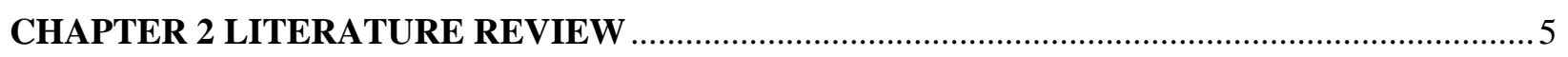

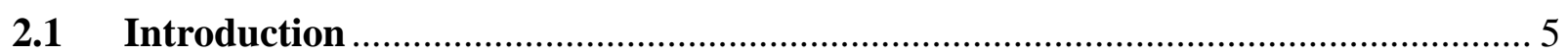

2.2 Generalized Quadratic Assignment Problem (GQAP) ......................................... 5

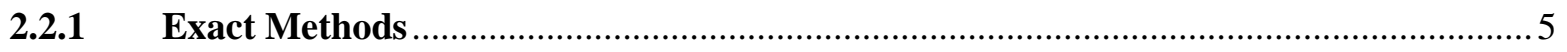

2.2.2 Heuristic Methods …………………………................................................

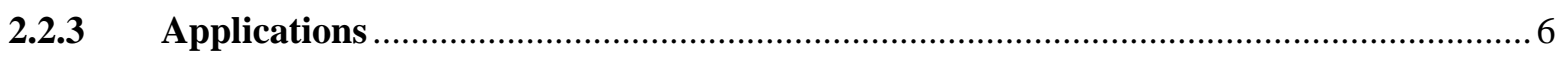

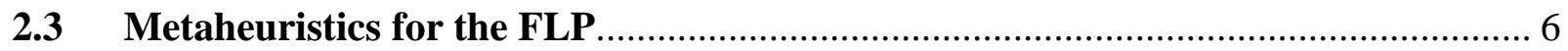

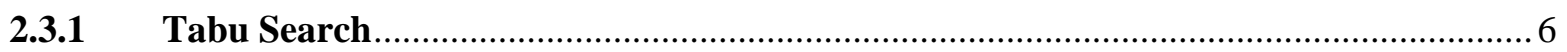

2.3.2 Simulated Annealing ......................................................................................... 6

2.4 Proposed Tabu Search (TS) Heuristic .................................................................. 7

2.5 Contributions of the Thesis to the GQAP Literature ……….................................... 7

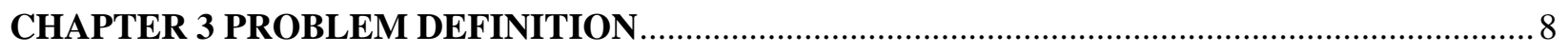

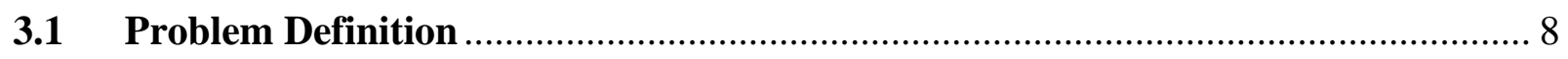

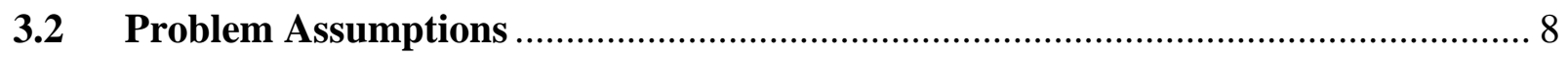

3.3 Mathematical Formulation ……………………............................................... 8

3.4 Combinatorial Optimization Problem (COP) Model ……….................................. 11

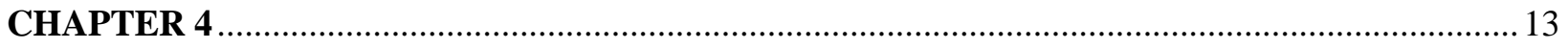

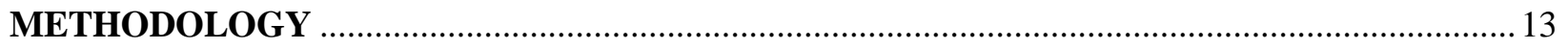

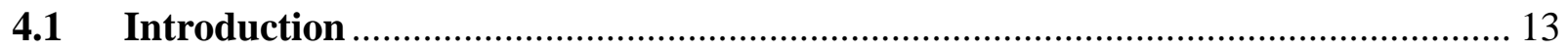

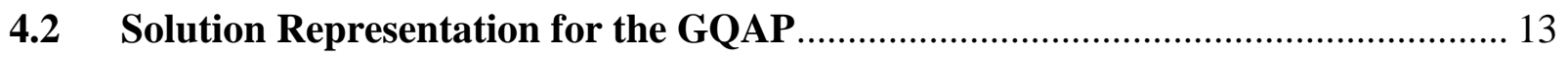

4.3 Construction Algorithm to Generate Initial Solution .............................................. 13

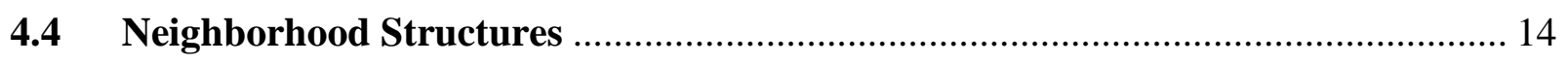

4.4.1 Drop/Add Neighborhood Structure ……………………………………………... 14

4.4.2 Pairwise Exchange Neighborhood Structure ……………………………………..... 15 
4.5 Determining Cost of the GQAP Solution ………….............................................. 16

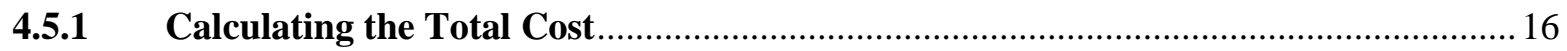

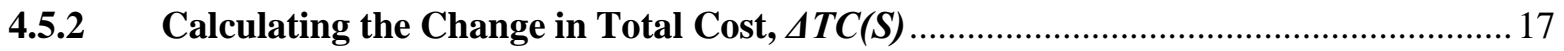

4.6 Steepest Descent Local Search Heuristic ……....................................................... 18

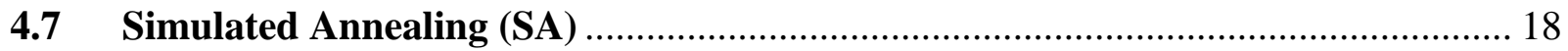

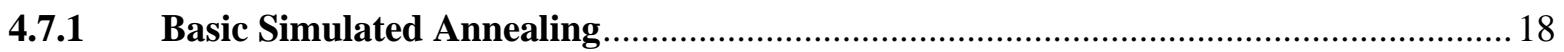

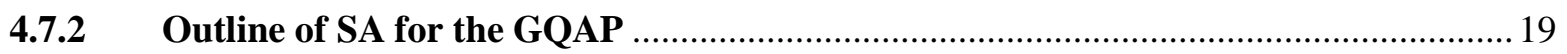

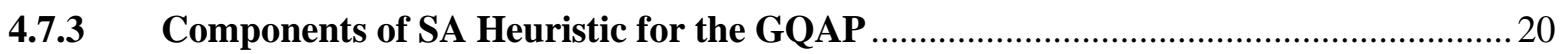

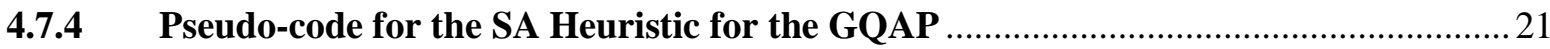

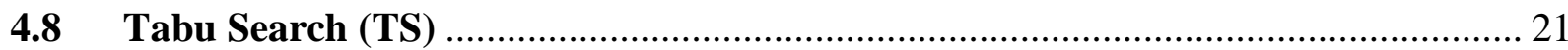

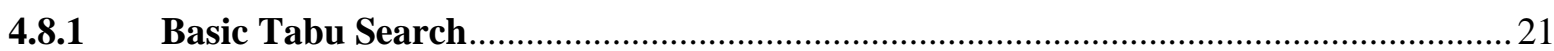

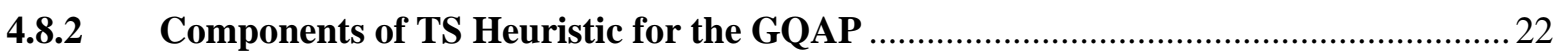

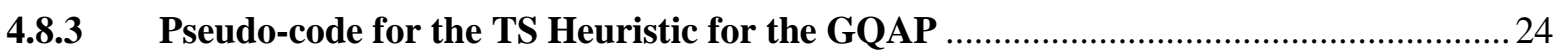

4.9 Comparison of Proposed Meta-heuristics.......................................................... 25

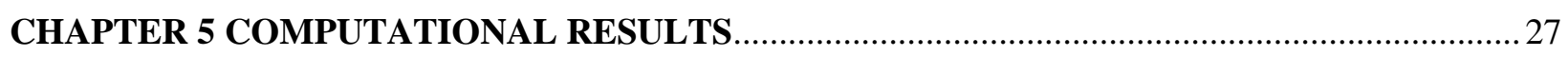

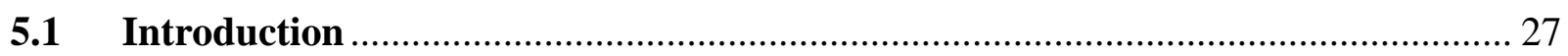

5.2 Computational Environment ………………………........................................ 27

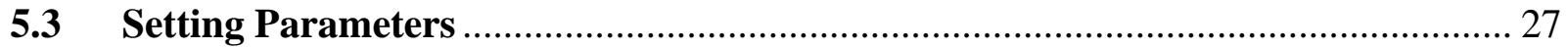

5.3.1 Parameters in Simulated Annealing (SA) Heuristics.................................................2

5.3.2 Parameters in Tabu Search (TS) Heuristics ............................................................. 35

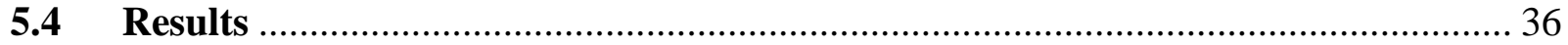

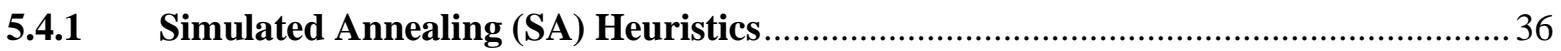

5.4.2 Tabu Search (TS) Heuristics............................................................................... 39

5.4 Comparison of Performances............................................................................ 42

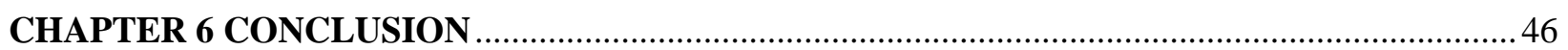

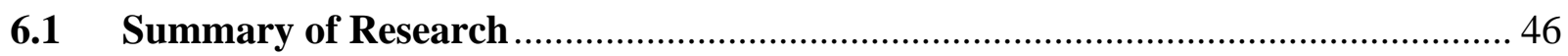

6.2 Recommendations for Future Research .............................................................. 46

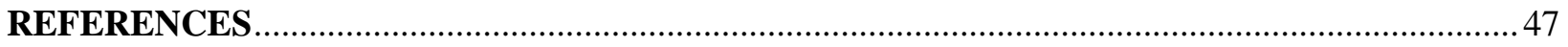




\section{LIST OF TABLES}

Table 4.1 Solutions obtained from drop/add operations on initial solution............................... 15

Table 4.2 Solutions obtained from pairwise exchange operations on initial solution ................. 16

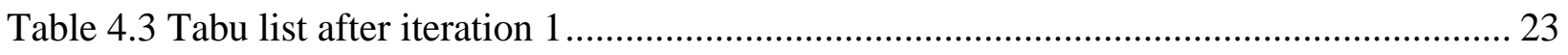

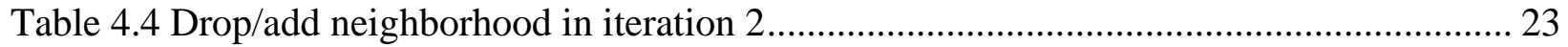

Table 4.5 Pairwise exchange neighborhood in iteration 2 ................................................. 23

Table 4.6 Tabu list after iteration 2 .............................................................................. 24

Table 5.1 Setting Initial Temperature for SA Heuristics ................................................ 28

Table 5.2 Results with $\mathrm{N}(\mathrm{t})=10 \%$ of the size of the neighborhood .................................... 29

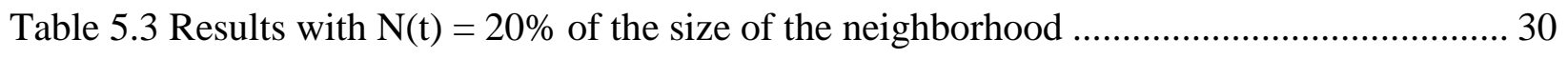

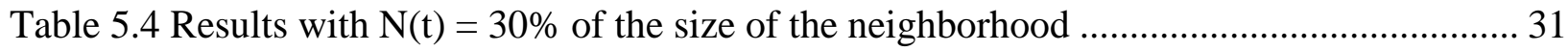

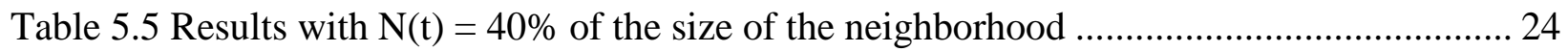

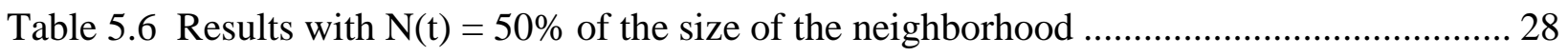

Table 5.7 Bar Chart with Percent Deviation for Different values of $\mathrm{N}(\mathrm{t})$................................ 34

Table 5.8 Results from SA Heuristics with Drop/Add Neighborhood ..................................... 36

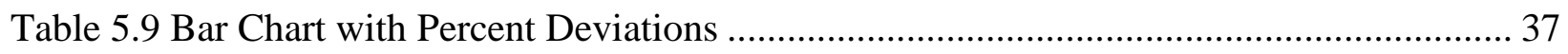

Table 5.10 Results from SA Heuristics with Both Neighborhoods ........................................ 38

Table 5.11a Results from TS Heuristics with Drop/Add Neighborhood (tabusize =0.02NBHD) 39

Table 5.11b Results from TS Heuristics with Drop/Add Neighborhood (tabusize =0.03NBHD) 40

Table 5.12 Results Obtained from TS Heuristics with Drop/Add where tabusize $=0.02 \mathrm{NBHD}$ or

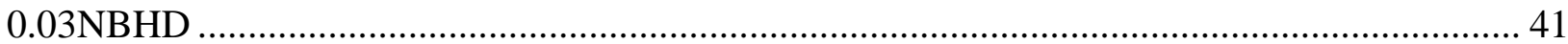

Table 5.13 Results from TS Heuristics with Both Neighborhoods ........................................ 42

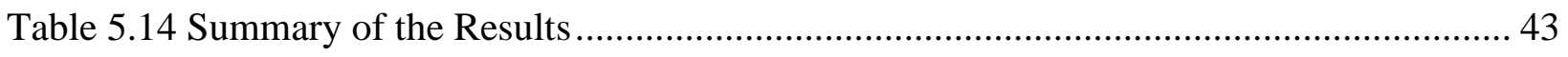

Table 5.15 Comparison of Results for the Proposed Heuristics .............................................. 44

Table 5.16 Comparison of Percent Deviations for the Proposed Heuristics.............................. 44 


\section{LIST OF FIGURES}

Figure 1.1 Different types of facility layouts ................................................................ 1

Figure 1.2 Simple classification of facility layout problems .............................................. 2

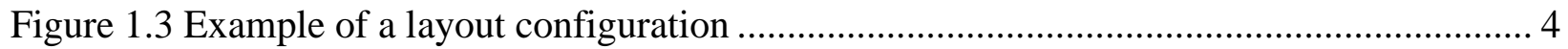

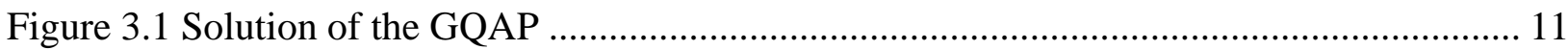




\section{CHAPTER 1 \\ INTRODUCTION}

\subsection{The Facility Layout Problem}

The facility layout problem (FLP) is defined as the placement of facilities in a plant area, with the aim of determining the most effective arrangement in accordance with some criteria or objectives under certain constraints, such as shape, size, orientation, and pick-up/drop-off point of the facilities.

The facility layout problems can be of different types. Different types of layouts are shown in Figure 1.1. Figure 1.1(a) shows a quadratic assignment problem (QAP) where all the departments are equal and only one machine is assigned to each location. Figure 1.1(b) shows an unequal area facility layout problem (UFLP) where we have rectangular grids and each machine is assigned a number of grids based on their area requirement.

\begin{tabular}{|c|c|c|c|}
\hline M15 & M5 & M11 & M2 \\
\hline M6 & M1 & M4 & M13 \\
\hline M14 & M8 & M12 & M7 \\
\hline M10 & M3 & M16 & M9 \\
\hline
\end{tabular}

(a) QAP (Discrete)

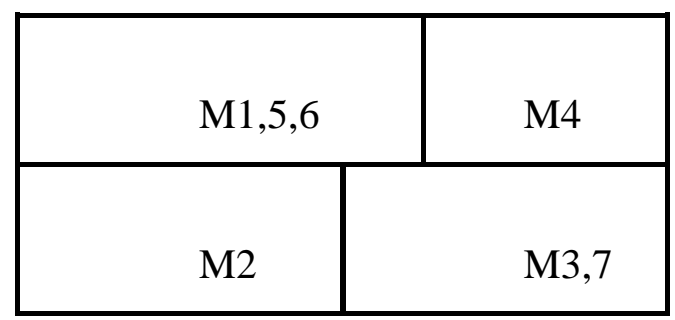

(c) GQAP (Discrete)

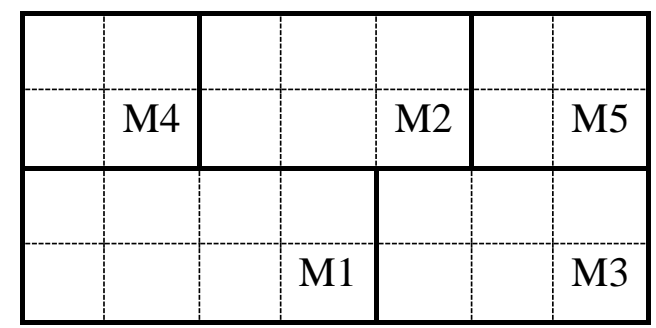

(b) UAFLP (Discrete)

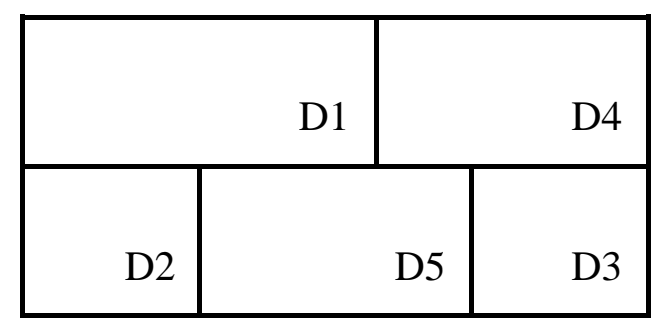

(d) Continuous

Figure 1.1: Different types of facility layouts

Figure 1.1(c) shows the generalized quadratic assignment problem (GQAP) where we have unequal area locations and more than one machine can be assigned to one location. Note, the first 
one (Figure 1.1(a)) deals with equal area location and the next two (Figures 1.1(b) \& (c)) deals with unequal area locations. All of these are discrete layout problems. However, Figure 1.1(d) shows a continuous UFLP where several departments with different shapes and areas are located on a rectangular floor.

A simple classification of facility layout problems can be shown by the figure below.

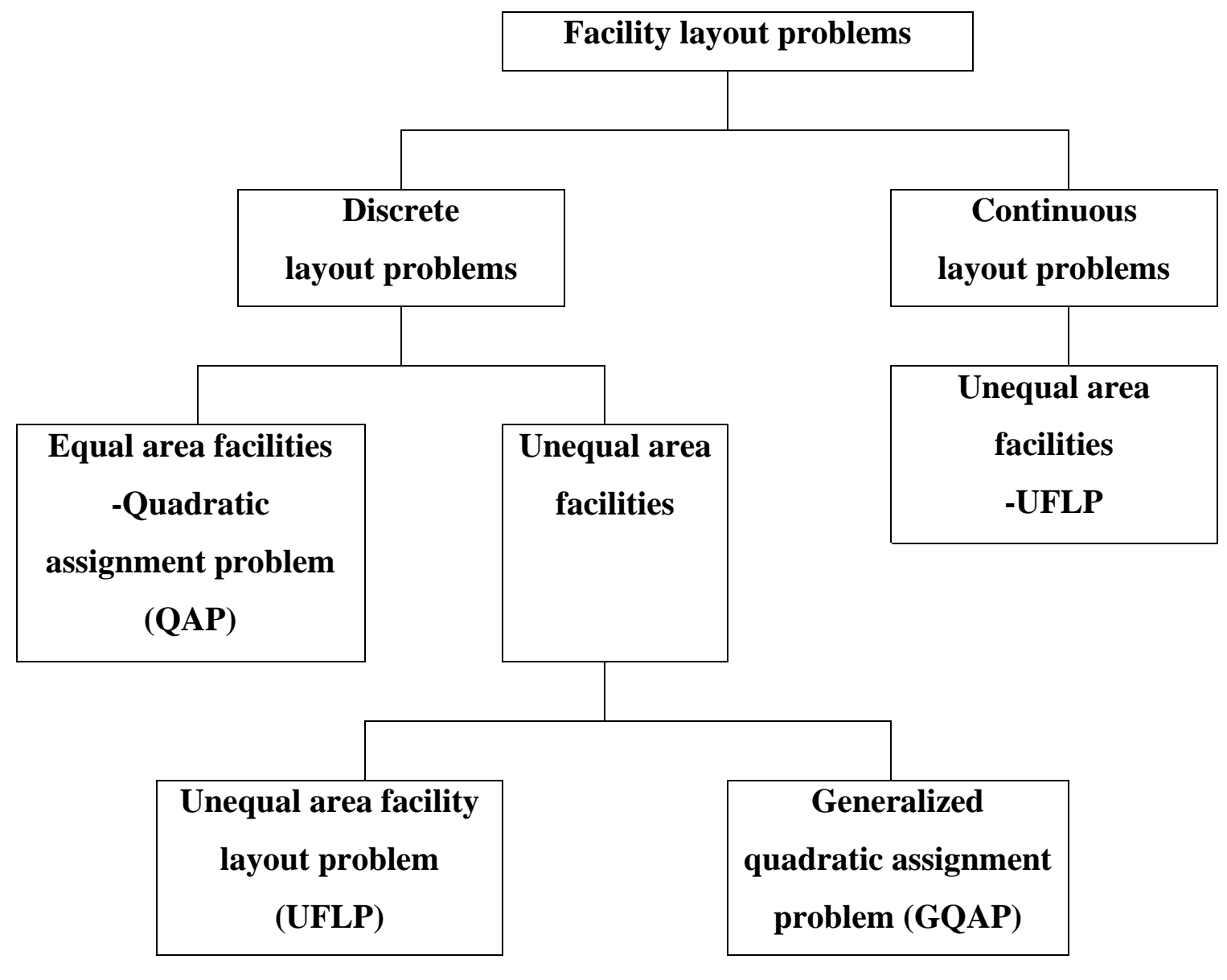

Figure 1.2: Simple classification of facility layout problems

In this research, the focus is on the GQAP (shown in Figure 1.1(c)) which is a discrete UFLP.

\subsection{The Importance of the Facility Layout Problem}

Facility layout dramatically influences the efficiency of material handling within a manufacturing system. Therefore, the facility layout is of great concern for manufacturers. An efficient facility 
layout will improve profit and productivity. Moreover, it has been estimated that materials handling cost is between 20 to $50 \%$ of the total operating cost, and effective facility layout planning can reduce the material handling costs by 10 to $30 \%$ (Tompkins et al., 2003).

Since customer demand is constantly changing, the material handling paths and layout of machines (or departments) are varied constantly. In other words, in order to ensure optimal performance of a facility, the layout should reflect changes to the system that may occur over time. Therefore, the FLP exists when either new plants are built or older plants are modified. Francis et al. (1992) proposed some reasons that may cause the modification of the layout of a facility:
a) Change of the product design.
b) The addition or deletion of a product from the product line.
c) Significant increase or decrease in the demand for a product.
d) Changes on the design of the process.
e) The replacement of equipment.
f) The adoption of new safety standards.
g) Bottlenecks in production.
h) Unexplainable delays and idle time.
i) Excessive temporary storage space.

Therefore, the FLP often occurs and exists for many different reasons. As a result, the facility layout may need to be modified constantly. The decision of the facility layout is made at the strategic level and has a long-lasting effect on the manufacturing system. Once the decision is made, changing the layout of the facility can be very costly. Some of the costs associated with the re-layout of a facility are cost of rearranging the machines, cost of purchasing or leasing equipment for rearranging the machines/department, and the cost associated with the loss of production. Therefore, the importance of the FLP is obvious.

\subsection{The Generalized Quadratic Assignment Problem}

The generalized quadratic assignment problem (GQAP) is the task of assigning a set of facilities to a set of locations such that the sum of the assignment and transportation costs is minimized. The facilities may have different space requirements, and the locations may have varying space 
capacities. Also, multiple facilities may be assigned to each location such that space capacity is not exceeded.

The material handling cost depends on the material flows between departments and the distances between their locations. The most commonly used distance measures for the FLP are rectilinear and Euclidean. The rectilinear distance between two points $\left(x_{1}, y_{1}\right)$ and $\left(x_{2}, y_{2}\right)$ is defined as $\left|x_{1}-x_{2}\right|+\left|y_{1}-y_{2}\right|$, and the Euclidean distance between the two points is defined as $\sqrt{\left(x_{1}-x_{2}\right)^{2}+\left(y_{1}-y_{2}\right)^{2}}$. Other distance measures can also be used to determine the distances between two departments. Nevertheless, in this research the rectilinear distance measure is used to determine the distance between two departments.

See Figure 1.3 for an example of a layout configuration with 4 locations. Notice the centroid of each location is given. For example, the centroid of location 4 is $(30,5)$. Using the rectilinear distance measure discussed above, the distance between locations 1 and 3 is 17.5 distance units and between locations 1 and 4 is 27.5 distance units.

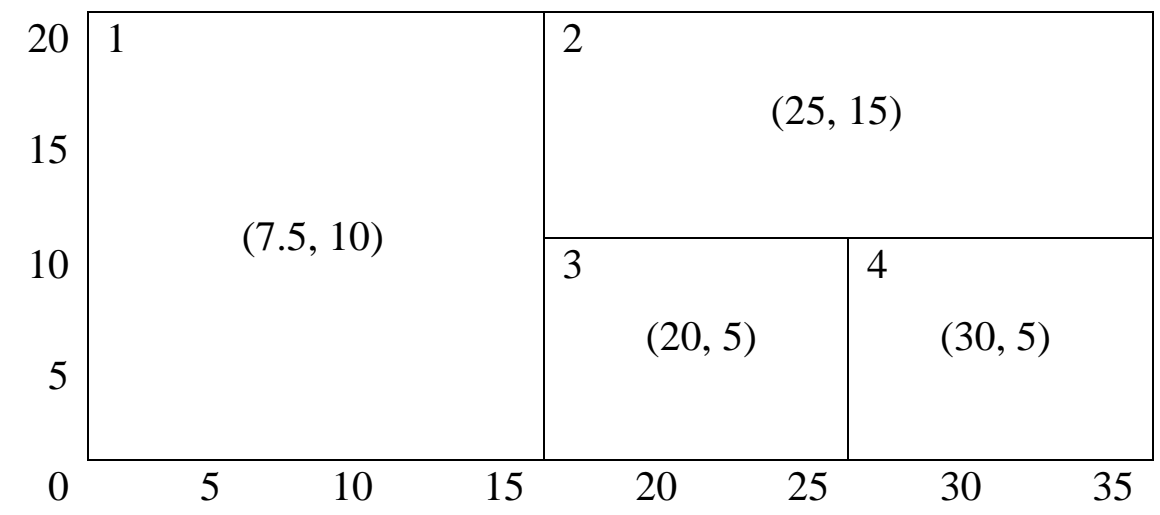

Figure 1.3: Example of a layout configuration

\subsection{Objective of the Thesis}

The objectives of this research are given as follows:

1. To develop a tabu search (TS) heuristic for the GQAP.

2. To develop a simulated annealing (SA) heuristic for the GQAP.

3. To test the performance of the heuristics by solving test problems taken from the literature.

4. To compare the performances of the two heuristics. 


\section{CHAPTER 2 \\ LITERATURE REVIEW}

\subsection{Introduction}

A good number of papers have been published on solving FLPs. But unlike QAP, GQAP literature is very limited. Exact methods cannot achieve optimal solutions for large scale problems. Heuristics are used to obtain good quality solutions in short computation times. However, the optimality of the solutions are not guaranteed by heuristic approaches. The literature review can be divided in two sections. The first one reviews exact methods and heuristic methods for GQAPs and their applications. And the second one is a brief review for tabu search (TS) and simulated annealing (SA) metaheuristics used in different types of FLPs.

\subsection{Generalized Quadratic Assignment Problem (GQAP)}

\subsubsection{Exact Methods}

Lee and Ma (2004) presented the first formulation for the GQAP. The authors presented three methods for the linearization of the formulation and a branch and bound algorithm to optimally solve the GQAP. Hahn et al. (2008) presented a new algorithm to optimally solve the GQAP. Pessoa et al. (2010) presented two exact algorithms for the GQAP which combine a previously proposed branch and bound scheme with a new Lagrangean relaxation procedure over a known RLT formulation.

\subsubsection{Heuristic Methods}

Cordeau et al. (2006) presented a linearization of the GQAP formulation as well as a memetic heuristic for the GQAP, which combines genetic algorithms (Holland,1975) and tabu search (Glover, 1986). Mateus et al. (2011) proposed several GRASP (greedy randomized adaptive search procedures) with path-relinking heuristics for the GQAP using different construction, local search, and path-relinking procedures (GRASP-PR). McKendall and Li (2017) presented an application of the GQAP for assigning a set of machines to a set of locations on the plant floor. Also, the authors developed a TS heuristic using different construction algorithms. McKendall (2008) presented three tabu search heuristics for a dynamic space allocation problem (DSAP) where each of the proposed heuristics outperformed the heuristics available in the literature for the DSAP. The DSAP is a layout-type problem that assigns items (project activity resources) to locations (either 
work or storage spaces) during a multi-period planning horizon such that the cost of rearranging the items is minimized.

\subsubsection{Applications}

Apart from facility layouts we have multiple application of the GQAP. Cordeau et al. (2006) presented an application which uses the GQAP to manage containers in a storage yard (assign $M$ container groups to $N$ storage areas). Cordeau et al. (2007) considered the service allocation problem as a GQAP with side constraints. Unal and Uysal (2014) used the GQAP to design a curriculum at a university. GQAP can also be used to assign maintenance activities and their required resources to workspaces and their idle resources to storage locations inside the reactor containment building during planned outages at a nuclear power plant (McKendall and Jaramillo, 2006).

\subsection{Metaheuristics for the FLP}

\subsubsection{Tabu Search}

In different types of FLPs, the TS has been widely used in optimizing material handling cost, utilization of space, minimization of re-layout cost for both single- and multi-objective problems (Abdinnour-Helm and Hadley, 2000; McKendall and Hakobyan, 2010; Scholz et al., 2009; Seo et al., 2006; Yang et al., 1999). TS is also deployed for optimizing single row fixed layout design problem (a special class of FLP) by Samarghandi and Eshghi (2010). Liang and Chao (2008) proposed a special different intensification and diversification strategies, which shows better convergence of searching to get proper arrangement of facilities. Improved TS algorithm with intensification, reconstruction and solution acceptance operation was proposed by Singh (2009), which gives comparative result with respect to some benchmark problems found in QAP-based FLP literature.

\subsubsection{Simulated Annealing}

SA is being used by the researchers in the field, starting from manufacturing cell design to multiobjective optimization of dynamic and static behaviour of FLP irrespective of whether there are equal or unequal sized facilities (Castillo and Peters, 2003; Dong et al., 2009; Ioannou, 2007; Sahin and Türkbey, 2009; Sahin et al., 2010; Souilah, 1995). Hybrid method of SA with TS (Sugiyono, 2006), SA and GA (Matsuzaki et al., 1999) helps to avoid high computational cost (Mir and Imam, 
2001) and improves the solution. McKendall et al., (2006) developed two SA algorithms for dynamic facility layout problems. A basic SA heuristic for the DFLP, and an SA heuristic with a look-ahead/look-back strategy. Gunawan et al. (2014) solved QAP using GRASP and improved the solution using a hybrid SA-TS algorithm. Privault et al. (1998) and Hamam et al. (2000) solved GAP using simulated annealing metahuristics.

\subsection{Proposed Tabu Search (TS) Heuristic}

As mentioned above in the GQAP literature review, a TS heuristic has been presented for the GQAP. In the TS heuristic presented in McKendall and Li (2017), the best admissible move is selected randomly among the top solutions at each iteration, to add randomness to diversify the search. This is a type of probabilistic TS heuristic and is not considered in this thesis. In other words, in the proposed TS heuristic the best admissible move is selected at each iteration. Also, McKendall and $\mathrm{Li}$ (2017) added even more diversification to their TS heuristic by generating a set of diverse initial solutions. In the proposed TS heuristic, only one initial solution is generated. For these reasons the proposed TS heuristic is more restricted. That is, the proposed TS heuristic presented in this thesis is deterministic.

\subsection{Contributions of the Thesis to the GQAP Literature}

The GQAP literature is very limited and there in no SA algorithm developed for the GQAP. Therefore, the contributions of this thesis, to the GQAP literature, is given as follows.

1. Development of a SA heuristic for the GQAP.

2. Improvement of the best found solutions for 3 test problems taken from the literature. 


\section{CHAPTER 3 \\ PROBLEM DEFINITION}

\subsection{Problem Definition}

There are $M$ machines to be installed in $N$ locations $(M \geq N)$ in a department. The machines have different space requirements and the locations have varying space capacities. Multiple machines can be assigned to each location such that space capacity is not exceeded. The problem is to assign the machines to the locations such that the total cost (i.e., the sum of installation cost and material handling costs) is minimized.

\subsection{Problem Assumptions}

The assumptions for the GQAP are as follows:

1. The layout configuration is given.

2. Machines and locations may be of unequal sizes.

3. The locations have rectangular areas.

4. Material flows between machines are given.

5. The distances between locations are known.

6. The area requirements for the machines are known.

7. The layout representation is discrete.

8. The objective is to minimize the sum of the installation and material handling costs.

\subsection{Mathematical Formulation}

The formulation of the GQAP from McKendall and Li (2017) is given below and is an adaptation of the model presented by Lee and Ma (2004).

$$
\begin{array}{ll}
\text { Minimize } \mathrm{z}= & \sum_{i=1}^{M} \sum_{k=1}^{N} a_{i k} x_{i k}+\sum_{i=1}^{M} \sum_{\substack{j=1 \\
j \neq i}}^{M} \sum_{k=1}^{N} \sum_{\substack{l=1 \\
l \neq k}}^{N} c_{i j k l} f_{i j} d_{k l} x_{i k} x_{j l} \\
\text { Subject to } \quad & \sum_{k=1}^{N} x_{i k}=1, \text { for } i=1, \ldots, M \\
& \sum_{i=1}^{M} r_{i} x_{i k}<=C_{k}, \text { for } k=1, \ldots, N \\
& x_{i k}=0 \text { or } 1, \text { for } i=1, \ldots, M \text { and } k=1, \ldots, N
\end{array}
$$


where,

$x_{i k}=1$ if machine $i$ is assigned to location $k$, and zero otherwise

$M=$ number of machines

$N=$ number of locations

$a_{i k}=$ cost of assigning machine $i$ to location $k$

$f_{i j}=$ units of materials transported from machine $i$ to machine $j$

$d_{k l}=$ distance from location $k$ to location $l$

$c_{i j k l}=$ unit cost per distance unit of moving materials from machine $i$, at location $k$, to machine $j$, at location $l$

$r_{i}=$ the space requirement of machine $i$

$C_{k}=$ the amount of space capacity available at location $k$

Objective function (1) minimizes the sum of the assignment (or installation) and material handling costs. Constraint set (2) ensures that each machine is assigned to only one location. Constraint set (3) ensures that the space capacity of each location is not exceeded, and the restrictions on the decision variables are given in (4).

The term in objective function (1) used to obtain material handling cost has a quadratic term (i.e. product of two variables). As a result, the mathematical formulation (1)-(4) is nonlinear and is called a nonlinear programming model. The model is linearized by substituting $w_{i k j l}$ for $x_{i k} x_{j l}$. Then, replace objective function (1) with

Minimize $z=\sum_{i=1}^{M} \sum_{k=1}^{N} a_{i k} x_{i k}+\sum_{i=1}^{M} \sum_{\substack{j=1 \\ j \neq i}}^{M} \sum_{k=1}^{N} \sum_{\substack{l=1 \\ l \neq k}}^{N} c_{i j k l} f_{i j} d_{k l} w_{i k j l}$

and add the following constraints,

$x_{i k}+x_{j l}-1 \leq w_{i k j l}$, for $i, j=1, \ldots, M$ where $j \neq i$ and $k, l=1, \ldots, N$ where $l \neq k$

$w_{i k j l} \geq 0$, for $i, j=1, \ldots, M$ where $j \neq i$ and $k, l=1, \ldots, N$ where $l \neq k$ 
As a result, the linearized model (i.e. a mixed integer linear programming model) for the GQAP consists of objective function (1a) subject to constraints (2)-(6).

For example, we can consider a GQAP instance where six machines are assigned to four locations on the plant floor (see Figure 1.3) and use the following data from McKendall and Li (2017).

$$
\begin{aligned}
a_{i k} & =\left[\begin{array}{cccc}
700 & 1600 & 1900 & 1400 \\
1300 & 1800 & 1700 & 800 \\
800 & 1400 & 3000 & 1100 \\
3000 & 800 & 700 & 1500 \\
1200 & 1500 & 1300 & 1800 \\
1700 & 800 & 1200 & 1100
\end{array}\right] \\
f_{i j} & =\left[\begin{array}{cccccc}
0 & 33 & 41 & 9 & 16 & 56 \\
0 & 0 & 49 & 91 & 78 & 23 \\
0 & 0 & 0 & 41 & 38 & 44 \\
0 & 0 & 0 & 0 & 6 & 17 \\
0 & 0 & 0 & 0 & 0 & 68 \\
0 & 0 & 0 & 0 & 0 & 0
\end{array}\right] \\
d_{k l} & =\left[\begin{array}{ccccc}
0 & 22.5 & 17.5 & 27.5 \\
22.5 & 0 & 15 & 15 \\
17.5 & 15 & 0 & 10 \\
27.5 & 15 & 10 & 0
\end{array}\right] \\
\mathrm{r}_{i} & =\left[\begin{array}{c}
90 \\
120 \\
100 \\
110 \\
110 \\
70
\end{array}\right] \\
\mathrm{C}_{k} & =\left[\begin{array}{cccc}
300 & 200 & 100 & 100
\end{array}\right] \text { and } c_{i j k l}=1 \text { for all } i, j, k, l
\end{aligned}
$$

Using the linearized mathematical model given above for the GQAP and the MPL/CPLEX software, the optimal solution is obtained for this problem: $x_{13}=x_{21}=x_{34}=x_{42}=x_{51}=x_{61}=1, z^{*}$ $=\$ 17,165$.

Interpretation of the solution is:

Machines 2, 5, and 6 are assigned to location 1.

Machines 4 is assigned to location 2.

Machines 1 is assigned to location 3. 
Machine 3 is assigned to location 4.

The solution of the GQAP instance is shown in Figure 3.1.

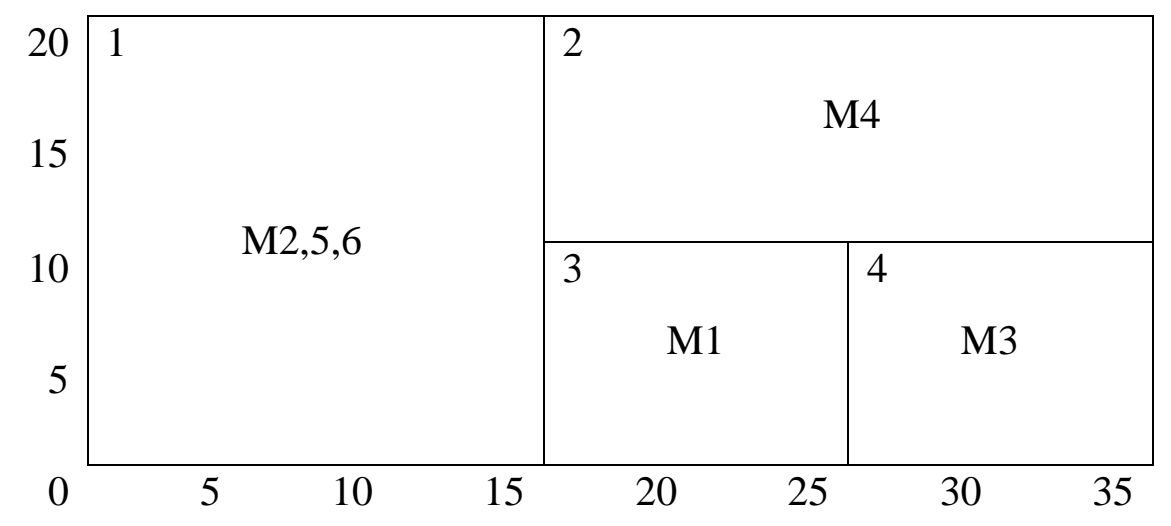

Figure 3.1: Solution of the GQAP Instance

The sum of total installation cost and total material handling cost is $z^{*}=\$ 17,165$ where total installation cost $=\sum_{i=1}^{6} \sum_{k=1}^{4} a_{i k} x_{i k}=\$ 8,000$ and total material handling cost $=\sum_{i=1}^{6} \sum_{\substack{j=1 \\ j \neq i}}^{6} \sum_{k=1}^{4} \sum_{\substack{l=1 \\ l \neq k}}^{4} c_{i j k l} f_{i j} d_{k l} w_{i k j l}=\$ 9,165$.

\subsection{Combinatorial Optimization Problem (COP) Model}

COPs are problems in which all or some of the decision variables are discrete. For the GQAP the COP model is given below:

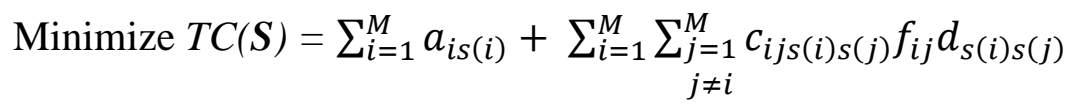

Subject to $\sum_{\forall i \text { s.t. } s(i)=\boldsymbol{k}} r_{i} \leq C_{k}$ for $k=1,2, \ldots, N$

where,

$\boldsymbol{J}=$ set of machines $\quad|\boldsymbol{J}|=M=$ number of machines

$\boldsymbol{K}=$ set of locations $\quad|\boldsymbol{K}|=N=$ number of locations

The solution representation is: $S=\{S(1), S(2), \ldots, S(M)\}$ where $S(i)=k$, i.e. machine $i$ is assigned to location $k$. 
Objective function (7) minimizes the total cost of the solution $S$. Constraint set (8) ensures that the capacity of each location is not violated.

The solution obtained in Section 3.3 for the GQAP instance can be represented as $S=(3,1,4,2$, $1,1)$ which indicates machines 2,5 , and 6 are assigned to location 1 , machines 4 is assigned to location 2, machines 1 is assigned to location 3, and machine 3 is assigned to location 4. Also, total cost of solution is $T C(S)=\$ 17,165$. 


\section{CHAPTER 4}

\section{METHODOLOGY}

\subsection{Introduction}

Metaheuristics can improve the performance of many other heuristics. Tabu search (Glover, 1986) is an aggressive metaheuristic that guides a local search out of local optimal while simulated annealing (Metropolis et al., 1953) uses a probabilistic approach to obtain the same end. In this thesis, a tabu search (TS) and a simulated annealing (SA) heuristic are developed to solve the COP model presented in the previous section to solve the GQAP.

\subsection{Solution Representation for the GQAP}

Recall, the solution representation for the GQAP is defined as follows:

$$
\begin{aligned}
& S=\{S(1), S(2), \ldots, S(M)\} \\
& \text { where } \\
& S=\text { solution of the GQAP } \\
& M=\text { number of machines } \\
& S(i)=k, \text { i.e. machine } i \text { is assigned to location } k .
\end{aligned}
$$

Next, a construction algorithm is presented for the GQAP.

\subsection{Construction Algorithm to Generate Initial Solution}

An initial solution for the GQAP is generated by a construction algorithm described in McKendall and $\mathrm{Li}$ (2017). It is given as follows.

Step 1: Initialize the capacities of the locations (i.e. $C=\{C(1), C(2), \ldots, C(N)\}$. Initialize the space requirements of the machines (i.e. $\boldsymbol{r}=\{r(1), r(2), \ldots, r(M)\}$.

Step 2: Sort machines in set $\boldsymbol{r}$ in descending order with respect to $r(i)$. Break ties based on smallest index (i.e. lowest machine number). Then put the sorted machine numbers in the eligible machine set (EMS). For example, if $\boldsymbol{r}=\{5,3,5\}$, then $r(1)=r(3)=5>3=r(2)$. Since index $1<3$, EMS $=$ $\{1,3,2\}$. 
Step 3a: Set $k=1$ where $k=$ location number.

Step 3b: If $k>N(N=$ number of locations), then go to step 5b. Else, go to position 1 of EMS (i.e. set $p=1$ ).

Step 4: Set $i=$ the machine in position $p$ of set EMS.

Step 5a: If $r(i) \leq C(k)$, then

1. Assign machine $i$ to location $k$ (i.e. set $s(i)=k$ ), and set $C(k)=C(k)-r(i)$;

2. Remove machine $i$ from EMS. If EMS is empty, then go to step 5b;

3. If $C(k)<r(i)$ for Last (i) in set EMS, then set $k=k+1$, and go to step 3b; Else, go to step 4. Else, set $p=p+1$, and go to step 4 .

Step 5b: Terminate algorithm. If EMS is empty, then display feasible solution $\boldsymbol{S}$. Else, display "No feasible solution!"

For the GQAP instance mentioned before, the solution generated using this construction algorithm is $\boldsymbol{S}_{\boldsymbol{0}}=(2,1,3,1,2,1)$ where total cost of this solution is $T C\left(\boldsymbol{S}_{\boldsymbol{0}}\right)=\$ 21,255$. Recall, the optimal solution obtained using MPL/CPLEX and the mathematical formulation (objective function (1a) subject to constraints (2)-(6)) presented in Chapter 3 is $S=(3,1,4,2,1,1)$ where $T C(S)=\$ 17,165$.

\subsection{Neighborhood Structures}

A neighborhood of a solution $\boldsymbol{S}_{\boldsymbol{0}}$ is a set of solutions that can be obtained from $\boldsymbol{S}_{\boldsymbol{0}}$ by a simple operation or move. In our proposed metaheuristics we used two types of neighborhood structures as defined in McKendall and Li (2017): drop/add and pairwise exchange.

\subsubsection{Drop/Add Neighborhood Structure}

Drop/add neighborhood considers a simply removal of an element and addition of an element to the set of solution variables. Using the notation taken from McKendall and $\mathrm{Li}$ (2017), the drop/add operation $\left(u, v: v^{\prime}\right)$ represents exchanging (dropping) location $v$ assigned to machine $u$ with (adding) location $v^{\prime}$. For example, if $\boldsymbol{S}_{\boldsymbol{0}}=\{2,1,3,1,2,1\}$, as obtained from using the construction algorithm presented above to solve the 6 machines 4 locations GQAP instance, then the drop/add operation $(1,2 ; 3)$ produces the solution $\{\underline{\mathbf{3}}, 1,3,1,2,1\}$. In other words, machine 1 assigned to location 2 is reassigned to location 3 . Since there are $M=6$ machines and $N=4$ locations, and 
each machine is already assigned to one location, there are $N-1=3$ possible drop/add operations for each machine. Therefore, there will be $M(N-1)=6(3)=18$ possible drop/add operations. See Table 4.1 (similar to Table 6 in McKendall and Li, 2017) for all possible drop/add operations on solution $\boldsymbol{S}_{\boldsymbol{0}}=(2,1,3,1,2,1)$.

Table 4.1 Solutions obtained from drop/add operations on initial solution

\begin{tabular}{|llll|llll|}
\hline$\#$ & Move & Solution & Cost & $\#$ & Move & Solution & Cost \\
\hline 1 & $(1,2 ; 1)$ & $(\underline{1}, 1,3,1,2,1)$ & infeasible & 10 & $(4,1 ; 2)$ & $(2,1,3, \underline{2}, 2,1)$ & infeasible \\
2 & $(1,2 ; 3)$ & $(\underline{3}, 1,3,1,2,1)$ & infeasible & 11 & $(4,1 ; 3)$ & $(2,1,3, \underline{3}, 2,1)$ & infeasible \\
3 & $(1,2 ; 4)$ & $(\underline{4}, 1,3,1,2,1)$ & $\$ 21,580$ & 12 & $(4,1 ; 4)$ & $(2,1,3, \underline{4}, 2,1)$ & infeasible \\
4 & $(2,1 ; 2)$ & $(2, \underline{2}, 3,1,2,1)$ & infeasible & 13 & $(5,2 ; 1)$ & $(2,1,3,1, \underline{1}, 1)$ & infeasible \\
5 & $(2,1 ; 3)$ & $(2, \underline{3}, 3,1,2,1)$ & infeasible & 14 & $(5,2 ; 3)$ & $(2,1,3,1, \underline{3}, 1)$ & infeasible \\
6 & $(2,1 ; 4)$ & $(2, \underline{4}, 3,1,2,1)$ & infeasible & 15 & $(5,2 ; 4)$ & $(2,1,3,1, \underline{4}, 1)$ & infeasible \\
7 & $(3,3 ; 1)$ & $(2,1, \underline{1}, 1,2,1)$ & infeasible & 16 & $(6,1 ; 2)$ & $(2,1,3,1,2, \underline{2})$ & infeasible \\
8 & $(3,3 ; 2)$ & $(2,1, \underline{2}, 1,2,1)$ & infeasible & 17 & $(6,1 ; 3)$ & $(2,1,3,1,2, \underline{3})$ & infeasible \\
9 & $(3,3 ; 4)$ & $(2,1, \underline{4}, 1,2,1)$ & $\$ 20,695$ & 18 & $(6,1 ; 4)$ & $(2,1,3,1,2, \underline{4})$ & $\$ 20,495$ \\
\hline
\end{tabular}

\subsubsection{Pairwise Exchange Neighborhood Structure}

Pairwise exchange considers interchanging or swapping two elements in the current solution. Using the notation taken from McKendall and Li (2017), the pairwise exchange operation $(u, v$; $\left.u^{\prime}, v^{\prime}\right)$ represents exchanging location $v$ assigned to machine $u$ with location $v$ ' assigned to machine $u^{\prime}$. If $\boldsymbol{S}_{\boldsymbol{0}}=\{\underline{2}, 1,3, \underline{1}, 2,1\}$, then the pairwise exchange operation $(1,2 ; 4,1)$ produces the solution $\{\underline{\mathbf{1}}, 1,3, \underline{\mathbf{2}}, 2,1\}$. In other words, machine 1 assigned to location 2 exchanges locations with machine 4 assigned to location 1 . Since two machines are swapping locations and there are $M=6$ machines, there are a combination of $M=6$ pick two i.e., $M(M-1) / 2=15$ possible pairwise exchange operations. If $v=v^{\prime}$, the solution does not change. Therefore, there are always less than $M(M-1) / 2$ pairwise exchange operations when a location has more than one machine assigned to it. See Table 4.2 (similar to Table 7 in McKendall and Li, 2017) for all possible pairwise exchange operations on solution $\boldsymbol{S}_{\boldsymbol{0}}=(2,1,3,1,2,1)$. 
Table 4.2 Solutions obtained from pairwise exchange operations on initial solution

\begin{tabular}{|llll|llll|}
\hline$\#$ Move & Solution & Cost & $\#$ & Move & Solution & Cost \\
\hline 1 & $(1,2 ; 2,1)$ & $(\underline{1}, \underline{2}, 3,1,2,1)$ & infeasible & 9 & $(2,1 ; 6,1)$ & $(2, \underline{1}, 3,1,2, \underline{1})$ & same as $S_{0}$ \\
2 & $(1,2 ; 3,3)$ & $(\underline{3}, 1, \underline{2}, 1,2,1)$ & infeasible & 10 & $(3,3 ; 4,1)$ & $(2,1, \underline{1}, \underline{3}, 2,1)$ & infeasible \\
3 & $(1,2 ; 4,1)$ & $(\underline{1}, 1,3, \underline{2}, 2,1)$ & infeasible & 11 & $(3,3 ; 5,2)$ & $(2,1, \underline{2}, 1, \underline{3}, 1)$ & infeasible \\
4 & $(1,2 ; 5,2)$ & $(\underline{2}, 1,3,1, \underline{2}, 1)$ & same as $S_{0}$ & 12 & $(3,3 ; 6,1)$ & $(2,1, \underline{1}, 1,2, \underline{3})$ & infeasible \\
5 & $(1,2 ; 6,1)$ & $(\underline{1}, 1,3,1,2, \underline{2})$ & infeasible & $\mathbf{1 3}$ & $\mathbf{( 4 , 1 ; 5 , 2 )}$ & $\mathbf{( 2 , 1 , 3 , ~}, \underline{\mathbf{1}}, \mathbf{1})$ & $\mathbf{\$ 1 8 , 0 5 0}$ \\
6 & $(2,1 ; 3,3)$ & $(2, \underline{3}, \underline{1}, 1,2,1)$ & infeasible & 14 & $(4,1 ; 6,1)$ & $(2,1,3, \underline{1}, 2, \underline{1})$ & same as $S_{0}$ \\
7 & $(2,1 ; 4,1)$ & $(2, \underline{1}, 3, \underline{1}, 2,1)$ & same as $S_{0}$ & 15 & $(5,2 ; 6,1)$ & $(2,1,3,1, \underline{1}, \underline{2})$ & infeasible \\
8 & $(2,1 ; 5,2)$ & $(2, \underline{2}, 3,1, \underline{1}, 1)$ & infeasible & & & & \\
\hline
\end{tabular}

\subsection{Determining Cost of the GQAP Solution}

\subsubsection{Calculating the Total Cost}

The total cost of the GQAP solution is the sum of assignment and material handling costs. The total assignment cost $(A(S))$ is calculated as follows:

$A(S)=\sum_{i=1}^{M} a_{i s(i)}$

where $a_{i k}=$ cost of assigning machine $i$ to location $k$

and the material handling cost $(M H(S))$ is calculated as follows:

$M H(S)=\sum_{i=1}^{M} \sum_{\substack{j=1 \\ j \neq i}}^{M} c_{i j s(i) s(j)} f_{i j} d_{s(i) s(j)}$

where

$f_{i j}=$ units of materials transported from machine $i$ to machine $j$

$d_{k l}=$ distance from location $k$ to location $l$

$c_{i j k l}=$ unit cost per distance unit of moving materials from machine $i$ (at location $k$ ) to machine $j$ (at location $l$ )

So, the total cost of the GQAP solution is calculated as follows: 


$$
\begin{aligned}
T C(S) & =A(S)+M H(S)=\sum_{i=1}^{M} a_{i s(i)}+\sum_{\substack{i=1 \\
j>i}}^{M} \sum_{\substack{j=1 \\
j>i}} c_{i j(i) s(j)} f_{i j} d_{s(i) s(j)} \\
& =\sum_{i=1}^{M} a_{i s(i)}+\sum_{i=1}^{M} \sum_{J=i+1}^{M} c_{i j s(i) s(j)} w_{i j} d_{s(i) s(j)}
\end{aligned}
$$

where $w_{i j}=f_{i j}+f_{j i}$

\subsubsection{Calculating the Change in Total Cost, $\triangle T C(S)$}

Once the objective function value (OFV) is obtained for the initial solution, $T C\left(S_{0}\right)$, the OFV of the neighboring solutions, $T C(S)$, can be obtained efficiently by calculating the change in total cost. The change in total cost is the sum of the change in assignment and material handling costs.

\subsubsection{1 $\triangle T C(S)$ in Drop/Add Neighborhood}

If a solution $\boldsymbol{S}$ is generated in the drop/add neighborhood of $\boldsymbol{S}_{\boldsymbol{0}}$ by performing the move $\left(u, v: v^{\prime}\right)$, the change in assignment cost $\Delta A_{u v}(S)$ is calculated as follows:

$\Delta A_{u v}(\mathbf{S})=a_{u v}-a_{u v \prime}$

And the change in material handling cost $\Delta M H_{u v}(\mathbf{S})$ is calculated as follows:

$$
\begin{gathered}
\Delta M H_{u v}(\mathbf{S})=\sum_{i=1}^{M} c_{i u s(i) v} w_{i u} d_{S(i) v}-\sum_{i=1}^{M} c_{i u s(i) v^{\prime}} w_{i u} d_{S(i) v^{\prime}} \\
=\sum_{i=1}^{M} c_{i u s(i) v} w_{i u}\left[d_{S(i) v}-d_{S(i) v^{\prime}}\right]
\end{gathered}
$$

So, the total change in cost after the drop/operation is:

$$
\begin{aligned}
\Delta T C= & T C\left(\boldsymbol{S}_{\boldsymbol{0}}\right)-T C(\boldsymbol{S})=\Delta A_{u v}(\boldsymbol{S})+\Delta M H_{u v}(\mathbf{S}) \\
& =\left(a_{u v}-a_{u v^{\prime}}\right)+\sum_{i=1}^{M} c_{i u s(i) v} w_{i u}\left[d_{S(i) v}-d_{S(i) v^{\prime}}\right]
\end{aligned}
$$

\subsubsection{2 $\Delta T C(S)$ in Pairwise Exchange Neighborhood}

If a solution $S$ is generated in the pairwise exchange neighborhood of $\boldsymbol{S}_{\mathbf{0}}$ by performing the move $\left(u, v: u^{\prime}, v^{\prime}\right)$, the change in assignment cost $\Delta A^{\prime}{ }_{u v}(\mathbf{S})$ is calculated as follows:

$\Delta A^{\prime}{ }_{u v}(S)=\left(a_{u v}+a_{u^{\prime} v^{\prime}}\right)-\left(a_{u v^{\prime}}+a_{u v v}\right)$

And the change in material handling cost $\Delta M H^{\prime}{ }_{u v}(\mathbf{S})$ is calculated as follows:

$$
\begin{aligned}
& \Delta M H^{\prime}{ }_{u v}(\mathbf{S})=\left[\sum_{i=1}^{M} c_{i u s(i) v} w_{i u} d_{S(i) v}+\sum_{i=1}^{M} c_{i u^{\prime} s(i) v^{\prime}} w_{i u^{\prime}} d_{S(i) v '}\right]-\left[\sum_{i=1}^{M} c_{i u s(i) v^{\prime}} w_{i u} d_{S(i) v^{\prime}}+\right. \\
& \left.\sum_{i=1}^{M} c_{i u^{\prime} s(i) v} w_{i u^{\prime}} d_{S(i) v}\right]
\end{aligned}
$$


$=\sum_{i=1}^{M} c_{i u s(i) v}\left[w_{i u}\left(d_{S(i) v}-d_{S(i) v^{\prime}}\right)+w_{i u^{\prime}}\left(d_{S(i) v^{\prime}}-d_{S(i) v}\right)\right]$

So, the total change in cost after the pairwise exchange operation is:

$\Delta T C=T C\left(\mathbf{S}_{\boldsymbol{0}}\right)-T C(\mathbf{S})=\Delta A^{\prime}{ }_{u v}(\mathbf{S})+\Delta M H^{\prime}{ }_{u v}(\mathbf{S})$

$=\quad\left[\left(a_{u v}+a_{u^{\prime} v^{\prime}}\right)-\left(a_{u v^{\prime}}+a_{u^{\prime} v}\right)\right]+\sum_{i=1}^{M} c_{i u s(i) v}\left[w_{i u}\left(d_{S(i) v}-d_{S(i) v^{\prime}}\right)+w_{i u^{\prime}}\left(d_{S(i) v^{\prime}}-\right.\right.$

$\left.\left.d_{S(i) v}\right)\right]$

\subsection{Steepest Descent Local Search Heuristic}

The local neighborhood search technique, called the steepest descent local search heuristic, is used in the proposed metaheuristics to get to the local optima. The steepest descent local search heuristic for the GQAP is given as follows.

Step 1: Construct a solution, $\boldsymbol{S}_{\boldsymbol{0}}=(S(1), S(2), \ldots, S(M))$, using the construction algorithm presented earlier and obtain its cost, $T C\left(\boldsymbol{S}_{\boldsymbol{0}}\right)$.

Step 2: Evaluate all feasible solutions obtained from all possible drop/add operations on $\boldsymbol{S}_{\boldsymbol{0}}$ and all possible pairwise exchange operations on $\boldsymbol{S}_{\boldsymbol{0}}$.

Step 3: Pick best operation/move which gives best feasible neighboring solution, $S$, with respect to total cost, $T C(\boldsymbol{S})$. If $T C(\boldsymbol{S})<T C\left(\boldsymbol{S}_{\boldsymbol{0}}\right)$, set $\boldsymbol{S}_{\boldsymbol{0}}=\boldsymbol{S}, T C\left(\boldsymbol{S}_{\boldsymbol{0}}\right)=T C(\boldsymbol{S})$, and go to step 2. Else, terminate heuristic and display local optimum $\boldsymbol{S}_{\boldsymbol{0}}$.

Next the SA heuristic is presented for the GQAP. Since SA is a probabilistic heuristic, it does not always converge to a local optimum solution. As a result, the solutions obtained from the proposed SA heuristic are either improved and/or verified as a local optimum using the steepest descent local search heuristic presented above.

\subsection{Simulated Annealing (SA)}

\subsubsection{Basic Simulated Annealing}

Simulated annealing (SA) algorithms model the process of annealing in solids to optimize complex functions or systems. In the physical world, annealing is accomplished by heating a solid to an elevated temperature and then allowing it to cool slowly enough so that the thermal equilibrium is maintained. Atoms in the material then assume a globally minimum energy state. SA algorithms have been successfully applied to a variety of problems. The algorithm starts with an initial 
solution and generates a new solution by changing one or more of the solution variables. The objective function is then evaluated for the new solution. While a better solution is always accepted there is a possibility that a worse solution may be accepted based on a probability function (Metropolis et al., 1953).

The change in energy is expressed as the change in objective function value (OFV), while the temperature is a control parameter that sets the probability of selection. In the general method, the temperature is held constant for a prescribed number of iterations to allow the system to gain "thermal equilibrium" and is then decreased in accordance with a cooling curve. As the temperature decreases, so does the probability that a worse solution will be accepted. This forces the algorithm to converge to an optimal, or near optimal, solution.

\subsubsection{Outline of SA for the GQAP}

SA heuristics starts with an initial solution $\boldsymbol{S}_{\boldsymbol{0}}$ and its cost $T C\left(\boldsymbol{S}_{\boldsymbol{0}}\right)$. Generates a neighboring solution $S$ randomly. The cost of the neighboring solution $T C(S)$ is obtained and compared to the cost of the initial solution $T C\left(S_{0}\right)$. If the cost of the neighboring solution is better (i.e., $T C(S)<T C\left(S_{0}\right)$ ) this solution becomes the best solution, and it is used as the starting solution at the next iteration (i.e., $\boldsymbol{S}_{\boldsymbol{0}}=\boldsymbol{S}$ ). Otherwise, the initial solution $\boldsymbol{S}_{\boldsymbol{0}}$ is used as the starting solution at the next iteration with respect to some probability. This process continues until a stopping criterion is reached. However, strategies, which use a local search technique that allowed accepting non-improving neighboring solutions, were considered and performed well. This is the idea behind the SA heuristic and other meta-heuristics. In other words, the idea of annealing is used to accept nonimproving moves (or neighboring solutions $\boldsymbol{S}$ ) to avoid getting trapped at a poor local optimum. In SA, the probability of accepting non-improving moves initially is high, but as the search proceeds (and the temperature is reduced), the probability of accepting non-improving moves reduces. An outline of a basic SA heuristic (McKendall et al., 2006) for combinatorial optimization problems is as follows:

1. Select settings for the heuristic parameters and generate an initial solution $\boldsymbol{S}_{\boldsymbol{0}}$ and its $\operatorname{cost} T C\left(\boldsymbol{S}_{\boldsymbol{0}}\right)$. This solution $\boldsymbol{S}_{\boldsymbol{0}}$ is defined as the current solution.

2. Obtain a neighboring solution $S$ of the current solution using a move or operation (e..g., drop add or pairwise exchange move). 
3. Obtain the cost of the neighboring solution $T C(S)$ and compare it to the cost of the current solution $T C\left(\boldsymbol{S}_{0}\right)$.

(a) If the cost is better (i.e., $T C(\boldsymbol{S})<T C\left(\boldsymbol{S}_{\boldsymbol{0}}\right)$ ), it is accepted as the current solution (i.e., $\boldsymbol{S}_{\boldsymbol{0}}$ $=S$ ).

(b) If the cost is worst or same (i.e., $T C(\boldsymbol{S}) \geq T C\left(\boldsymbol{S}_{0}\right)$ ), then it is accepted as the current solution (i.e., $S_{\boldsymbol{0}}=S$ ) with some probability. Otherwise, keep the current solution $\boldsymbol{S}_{\boldsymbol{0}}$.

4. Update counters and parameters, and repeat steps $2-4$, until an appropriate stopping criterion is reached.

\subsubsection{Components of SA Heuristic for the GQAP}

\subsubsection{Initial Temperature $T(0)$}

Initial Temperature controls the probability that a non-improving move will be accepted. The initial temperature is determined such that the probability of accepting a neighboring solution $S$ with a cost of $x \%$ above the cost of the initial solution $S_{0}$ is $P(\Delta T C)$ where $\triangle T C=T C\left(S_{0}\right)-T C(S)$. The probability $P(\Delta T C)=\exp (-\Delta T C / T(t))$. For iteration $1, T(t)=T(0)$, which gives the equation: $T(0)=-\Delta T C / \ln (P(\Delta T C))=\frac{-\left(\frac{x}{100}\right) T C\left(S_{0}\right)}{\ln (P(\Delta T C)}$.

\subsubsection{Number of iterations at each temperature $N(t)$}

This is the number of iterations at each temperature which can be static or dynamic.

\subsubsection{Cooling Ratio $\alpha$}

Cooling ratio specifies the fraction of the current temperature $T(t)$ that will be used as the next temperature once we have attempted a number of iterations equal to $N(t)$. The cooling schedule is $T(t)=\alpha T(t-1)$ where $0<\alpha<1$.

\subsubsection{Stopping Criterion}

The stopping criterion for the proposed SA heuristic is the minimum allowable temperature. The heuristic terminates when the temperature reaches a value close to zero. Other commonly used stopping criteria are computational time, maximum number of iterations performed and maximum number of consecutive iterations without an improvement. The detailed settings of the heuristic parameters are explained in Chapter 5. 


\subsubsection{Pseudo-code for the SA Heuristic for the GQAP}

The pseudo-code for the SA heuristic with the different neighborhoods (i.e., drop/add, pairwise exchange neighborhoods) for the GQAP is given below.

Step 1: Obtain initial solution $S_{\boldsymbol{0}} \in \boldsymbol{X}$ where $\boldsymbol{X}$ is the solution space (set of all feasible solutions). Set Best_sol $=\boldsymbol{S}_{\boldsymbol{0}}$ and Best_cost $=T C\left(\boldsymbol{S}_{\boldsymbol{0}}\right)$.

Step 2: Select initial temperature $T(0), T>0$, the cooling ratio $\alpha$, attempted number of moves at each temperature, and the minimum allowable temperature.

Step 3: Set temperature counter $t=0$, iteration counter at current temperature $n=0$.

Step 4: Randomly choose a neighborhood structure between drop/add and pairwise exchange.

Step 5: Randomly generate a neighbor of $\boldsymbol{S}_{\boldsymbol{0}}$. For drop/add neighborhood, randomly select a machine $u$ and a location $v^{\prime}$, then assign machine $u$ to location $v^{\prime}$. For pairwise neighborhood, randomly select two machines $u$ and $v \neq u$ and exchange their locations.

Step 6: Obtain the change in the total cost, $\triangle T C=T C\left(S_{0}\right)-T C(S)$.

Step 7: If $\Delta T C>0$, then $\boldsymbol{S}_{\boldsymbol{0}}=\boldsymbol{S}$. If $T C\left(\boldsymbol{S}_{\boldsymbol{0}}\right)<$ Best_cost, update Best_sol and Best_cost such that Best_sol $=\boldsymbol{S}_{\boldsymbol{0}}$ and Best_cost $=T C\left(\boldsymbol{S}_{\boldsymbol{0}}\right)$.

Else if $\Delta T C<0$ and $\exp (\Delta T C / T)>$ rand $(0,1)$, then $\boldsymbol{S}_{\boldsymbol{0}}=\boldsymbol{S}$. Else keep $\boldsymbol{S}_{\boldsymbol{0}}$.

Set $k=k+1$ and $n=n+1$. Repeat steps 4-7 until $n=N(t)$ or until reaching stopping criterion.

If $n=N(t)$ go to step 8. If stopping criterion is reached go to step 9 .

Step 8: Set $t=t+1, T=T(t)=\alpha T(t-1), n=0$. Go to step 4.

Step 9: Use steepest descent local search heuristic on the best solution obtained.

Note: For SA heuristic with only drop/add neighborhood, insteps 4 and 5, consider and generate a neighbor of $\boldsymbol{S}_{\boldsymbol{0}}$ using a drop/add move only.

\subsection{Tabu Search (TS)}

\subsubsection{Basic Tabu Search}

Tabu search (TS) has been shown to be effective on a wide variety of classical optimization problems, such as graph colouring and travelling salesman problems, and has also been applied to 
practical problems such as scheduling and electronic circuit design. The method uses aspiration levels and tabu restrictions which will be discussed below. The search method in the proposed implementation is a steepest descent algorithm. It explores the set of feasible solutions by moving from a solution to its best neighbor (the solution with the best objective function value in a neighborhood), even if this results in a worst objective function value. The short-term memory contains representations of certain numbers of recent moves (formed as a tabu list). These moves are forbidden or declared as tabu restricted for a number of iterations which is known as the tabu duration. As the search progresses the list is updated on a first in-last out basis so that the list remains a fixed size. It is the short-term memory and the notion of tabu restriction that provide the capability to escape local optima. The algorithm is run for a prescribed number of iterations or a prescribed duration of time and then the best solution generated so far is chosen. In this research, a TS heuristic is presented similar to the TS used in McKendall and Li (2017). The different between two will be discussed below.

\subsubsection{Components of TS Heuristic for the GQAP}

\subsubsection{Tabu List}

After performing one iteration of the TS heuristic, the best admissible move (discussed below) is defined as tabu (tabu restricted) for a certain number of iterations (called tabu length or duration). The tabu list is used to keep track of the tabu moves. The best admissible move is either a nontabu move that generates the lowest cost feasible solution out of all the possible non-tabu moves at the current iteration or a tabu move that produces the lowest cost feasible solution found thus far, considering all the iterations performed.

If the best feasible solution in the neighborhood of the current solution $\boldsymbol{S}_{\boldsymbol{0}}$ is $\boldsymbol{S}$, and $\boldsymbol{S}$ is obtained by the drop/add move $\left(u, v ; v^{\prime}\right)$, then this move is tabu restricted for a certain number of iterations, called tabu list size (tabusize). The tabu status and tabu list size of each move are maintained in the tabu list structure $t a b u[u][v]$. For example, we can consider the results obtained in the first iteration (see Table 4.1 and Table 4.2). In iteration 1, the best move is the exchange move $(4,1 ; 5$, 2 ), which yields the best feasible solution $\boldsymbol{S}_{\boldsymbol{0}}=(2,1,3,2,1,1)$ (with lowest OFV of \$18,050) in the neighborhood of the current solution $\boldsymbol{S}_{\boldsymbol{0}}$. Therefore, the move $(4,1 ; 5,2)$ is tabu until iter = tabusize + iter and update tabu list: $\operatorname{tabu}[4][1]=\operatorname{tabu}[5][2]=$ tabusize + iter. That is, the move, exchanging location 1 assigned to machine 4 with location 2 assigned to machine 5 , can be 
performed again when iter $>$ tabusize + iter i.e. iter $>$ tabusize +1 since current iteration is iteration 1 (iter $=1$ ). If we consider a tabu size of 6 , after iteration 1 , the tabu list will look like Table 4.3.

Table 4.3 Tabu list after iteration 1

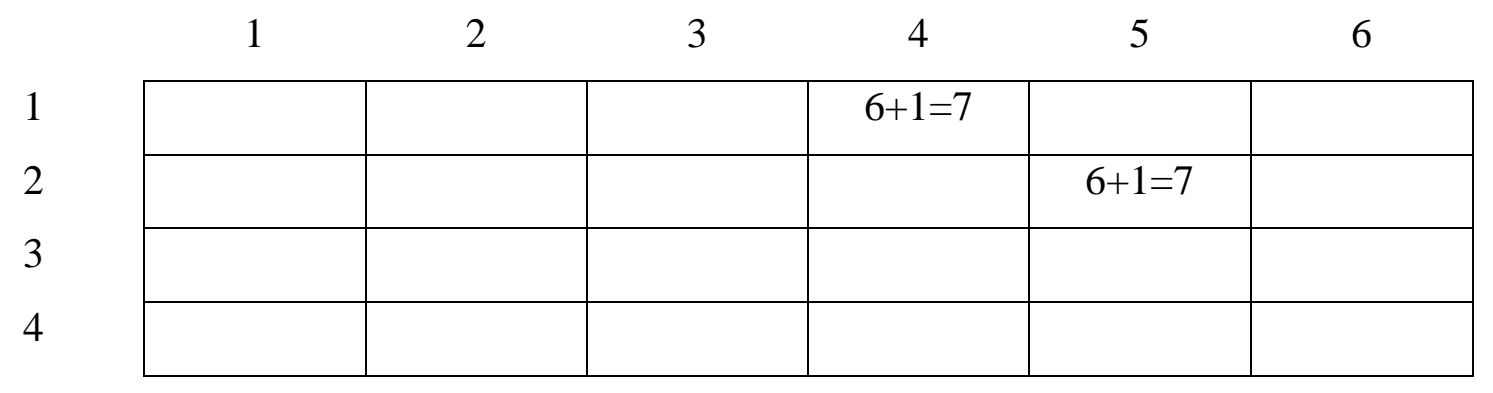

Table 4.4 Drop/add neighborhood in iteration 2

\begin{tabular}{|c|c|c|c|c|c|c|c|}
\hline \# & Move & Solution & Cost & $\#$ & Move & Solution & Cost \\
\hline 1 & $(1,2 ; 1)$ & $(1,1,3,2,1,1)$ & infeasible & 10 & $(4,2 ; 1)$ & $(2,1,3, \underline{1}, 1,1)$ & infeasible \\
\hline 2 & $(1,2 ; 3)$ & $(\underline{3}, 1,3,2,1,1)$ & infeasible & 11 & $(4,2 ; 3)$ & $(2,1,3, \underline{3}, 1,1)$ & infeasible \\
\hline 3 & $(1,2 ; 4)$ & $(\underline{4}, 1,3,2,1,1)$ & $\$ 18,305$ & 12 & $(4,2 ; 4)$ & $(2,1,3, \underline{4}, 1,1)$ & infeasible \\
\hline 4 & $(2,1 ; 2)$ & $(2, \underline{2}, 3,2,1,1)$ & infeasible & 13 & $(5,1 ; 2)$ & $(2,1,3,2, \underline{2}, 1)$ & infeasible \\
\hline 5 & $(2,1 ; 3)$ & $(2, \underline{3}, 3,2,1,1)$ & infeasible & 14 & $(5,1 ; 3)$ & $(2,1,3,2, \underline{3}, 1)$ & infeasible \\
\hline 6 & $(2,1 ; 4)$ & $(2, \underline{4}, 3,2,1,1)$ & infeasible & 15 & $(5,1 ; 4)$ & $(2,1,3,2, \underline{4}, 1)$ & infeasible \\
\hline 7 & $(3,3 ; 1)$ & $(2,1, \underline{1}, 2,1,1)$ & infeasible & 16 & $(6,1 ; 2)$ & $(2,1,3,2,1,2)$ & infeasible \\
\hline 8 & $(3,3 ; 2)$ & $(2,1, \underline{2}, 2,1,1)$ & infeasible & 17 & $(6,1 ; 3)$ & $(2,1,3,2,1, \underline{3})$ & infeasible \\
\hline 9 & $(3,3 ; 4)$ & $(2,1, \underline{4}, 2,1,1)$ & $\$ 17,460$ & 18 & $(6,1 ; 4)$ & $(2,1,3,2,1, \underline{4})$ & $\$ 19,075$ \\
\hline
\end{tabular}

Table 4.5 Pairwise exchange neighborhood in iteration 2

\begin{tabular}{|cccc|cccc|}
\hline$\#$ & Move & Solution & Cost & $\#$ & Move & Solution & Cost \\
\hline 1 & $(1,2 ; 2,1)$ & $(\underline{1}, \underline{2}, 3,2,1,1)$ & infeasible & 9 & $(2,1 ; 6,1)$ & $(2, \underline{1}, 3,2,1, \underline{1})$ & same as $S_{0}$ \\
2 & $(1,2 ; 3,3)$ & $(\underline{3}, 1, \underline{2}, 2,1,1)$ & infeasible & 10 & $(3,3 ; 4,2)$ & $(2,1, \underline{2}, \underline{3}, 1,1)$ & infeasible \\
3 & $(1,2 ; 4,2)$ & $(\underline{2}, 1,3, \underline{2}, 1,1)$ & same as $S_{0}$ & 11 & $(3,3 ; 5,1)$ & $(2,1, \underline{1}, 2, \underline{3}, 1)$ & infeasible \\
4 & $(1,2 ; 5,1)$ & $(\underline{1}, 1,3,2, \underline{2}, 1)$ & infeasible & 12 & $(3,3 ; 6,1)$ & $(2,1, \underline{1}, 2,1, \underline{3})$ & infeasible \\
5 & $(1,2 ; 6,1)$ & $(\underline{1}, 1,3,2,1, \underline{2})$ & infeasible & 13 & $(4,2 ; 5,1)$ & $(2,1,3, \underline{1}, \underline{2}, 1)$ & $\$ 21,255$ \\
6 & $(2,1 ; 3,3)$ & $(2, \underline{3}, \underline{1}, 2,1,1)$ & infeasible & 14 & $(4,2 ; 6,1)$ & $(2,1,3, \underline{1}, 1, \underline{2})$ & infeasible \\
7 & $(2,1 ; 4,2)$ & $(2, \underline{2}, 3, \underline{1}, 1,1)$ & infeasible & 15 & $(5,1 ; 6,1)$ & $(2,1,3,2, \underline{1}, \underline{1})$ & same as $S_{0}$ \\
8 & $(2,1 ; 5,1)$ & $(2, \underline{1}, 3,2, \underline{1}, 1)$ & same as $S_{0}$ & & & & \\
\hline
\end{tabular}


Similarly, in iteration 2 (see Tables 4.4 and 4.5 for all possible drop/add and pairwise exchange moves for current solution $\left.\boldsymbol{S}_{\boldsymbol{0}}=(2,1,3,2,1,1)\right)$, since the best admissible move is the drop/add move $(3,3 ; 4)$, tabu[3][3] $=$ tabusize + iter $=$ tabusize +2 . So the tabu list will be updated accordingly (see Table 4.6).

Table 4.6 Tabu list after iteration 2

\begin{tabular}{l|l|l|l|l|l|l|}
\multicolumn{2}{c}{1} & \multicolumn{2}{c}{3} & \multicolumn{2}{c}{4} & \multicolumn{1}{c|}{6} \\
\cline { 2 - 7 } \\
\cline { 2 - 7 } \\
\cline { 2 - 7 } 3 \\
\cline { 2 - 7 } \\
\cline { 2 - 7 } & & & & $6+1=7$ & & \\
\cline { 2 - 7 } & & & & & & \\
\cline { 2 - 7 } & & & & & & \\
\hline
\end{tabular}

\subsubsection{Aspiration Criterion}

The aspiration criterion is a short-term memory strategy used in the tabu search heuristic to override the tabu restriction. If a move is tabu in the tabu list and produces a solution with a better objective function value compared to that of the best found solution so far, the move is admissible. In other words, the tabu restriction is overridden even though the move is tabu.

\subsubsection{Stopping Criterion}

The stopping criterion for the proposed TS heuristic is computational time. This stopping criterion is used to compare the performances of the proposed TS heuristics with the proposed SA heuristics. Other commonly used stopping criteria are maximum number of iterations performed and maximum number of consecutive iterations without an improvement. The detailed settings of the heuristic parameters are explained in Chapter 5.

\subsubsection{Pseudo-code for the TS Heuristic for the GQAP}

The TS heuristic proposed here is similar to the TS heuristic proposed in McKendall and Li (2017). The proposed TS heuristics consider the drop/add neighborhood structure by itself, and both the drop/add and pairwise exchange neighborhood structures together. However, in McKendall and Li (2017) the authors consider the drop/add and pairwise exchange neighborhood structures together. The pseudo-code for the TS heuristic with the different neighborhoods (i.e., drop/add, pairwise exchange neighborhoods) for the GQAP is given below. 
The pseudo-code for the TS heuristic with both drop/add and pairwise exchange neighborhood for the GQAP is given below.

Step 1: Initialize parameters and counters: Set $t a b u[u][v]=0$, for all $u=1, \ldots, M$ and $v=1, \ldots$, $N$. Set tabusize, where tabusize $=$ tabu tenure length. Set MaxTime, where MaxTime = maximum computational time for the problem before terminating heuristic. Set iter $=0$, where iter $=$ iteration number.

Step 2: Obtain an initial solution $\boldsymbol{S}_{\boldsymbol{0}}=(S(1), S(2), \ldots, S(M))$ using the construction algorithm presented in Section 4.3. Obtain $T C\left(\boldsymbol{S}_{0}\right)$ using objective function.

Step 3: Set $S^{*}=S_{0}$, where $S^{*}$ is the best solution found thus far, and set $T C\left(S^{*}\right)=T C\left(S_{0}\right)$;

Step 4: Set iter $=$ iter +1 ; Find the best admissible move, which gives best feasible solution $(\boldsymbol{S})$ in neighborhood $\boldsymbol{S}_{\boldsymbol{0}}$. Find best feasible solution $(\boldsymbol{S})$ by considering all possible drop/add and pairwise exchange operations on $\boldsymbol{S}_{\boldsymbol{o}}$.

Step 5: Set $\boldsymbol{S}_{\boldsymbol{0}}=\boldsymbol{S}$ and $T C\left(\boldsymbol{S}_{\boldsymbol{0}}\right)=T C(\boldsymbol{S})$; If $T C\left(\boldsymbol{S}_{\boldsymbol{0}}\right)<T C\left(\boldsymbol{S}^{*}\right)$, set $\boldsymbol{S}^{*}=\boldsymbol{S}_{\boldsymbol{0}}$ and $T C\left(\boldsymbol{S}^{*}\right)=T C\left(\boldsymbol{S}_{\boldsymbol{0}}\right)$.

Step 6: Update tabu list as either tabu[u] $[v]=$ iter + tabusize, if drop/add operation $\left(u, v ; v^{\prime}\right)$ gives best admissible move or tabu[u][v] = tabu[u'][v'] = iter + tabusize, if pairwise exchange operation $\left(u, v ; u^{\prime}, v^{\prime}\right)$ gives best admissible move.

Step 7: Stopping criterion: If iter < MaxTime, go to Step 4. Else, terminate the heuristic and return solution $S^{*}$ and total cost of solution $T C\left(S^{*}\right)$.

Note: For the TS heuristic with only drop/add neighborhood, consider all possible drop/add moves only in step 4.

\subsection{Comparison of Proposed Meta-heuristics}

In TS heuristic the tabu list provides a short-term memory and it is a very deterministic heuristic. That is, the best admissible move is selected at each iteration. To diversify the search, the best admissible move in, say, the top $10-25 \%$ can be selected at each iteration to add randomness to the heuristic. This is called the probabilistic TS heuristic. Although this heuristic exists in the literature, it is not considered in this thesis. On the other hand, SA is very stochastic in nature (i.e., 
at each iteration, a move is randomly selected). As a result, an extremely deterministic heuristic (i.e., TS) and a very stochastic heuristic (i.e., SA) is developed for the proposed GQAP.

After solving problems of different types and sizes, the results obtained from the two diverse heuristics are compared. To compare the performance of the heuristics, the objective function value (total cost) as well as time taken to solve the problems should be considered. The comparisons are discussed in Section 5.4. 


\section{CHAPTER 5 \\ COMPUTATIONAL RESULTS}

\subsection{Introduction}

The proposed simulated annealing (SA) and tabu search (TS) heuristics were tested on a set of 21 test problems given by Cordeau et. al. (2006). This problem set was also used in other literature for the GQAP (Mateus et. al., 2011 and McKendall and Li, 2017). The set of test problems contains problem instances with 20,30, 35, 40, and 50 machines with locations ranging between 6 and 20 such that the percentages of location area required (i.e. tightness of the capacity constraint) ranges between 35 and 95. As mentioned previously, the proposed SA and TS heuristics used one of the construction algorithms presented by McKendall and Li (2017) to generate the initial solutions (see Section 4.3).

\subsection{Computational Environment}

The proposed SA and TS heuristics were programmed using MATLAB and executed on a 3.30 GHz Intel Core i5 CPU with 16 GB of RAM under the Microsoft Windows Operating System.

\subsection{Setting Parameters}

\subsubsection{Parameters in Simulated Annealing (SA) Heuristics}

Preliminary analyses are performed to determine the SA heuristic parameter settings.

1. Initial Temperature, $T(0)$

McKendall et. al. (2006) accepted a non-improving neighboring solution (with $25 \%$ probability) with a cost of $10 \%$ above the cost of the initial solution to set the initial temperature for a SA heuristic for a dynamic facility layout problem. Privault et. al. (1998) accepted 80\% of the candidate solutions (i.e., non-improving feasible or infeasible neighboring solutions) for a type of assignment problem. Note, in this research, only feasible solutions are candidate solutions.

In a preliminary experiment, three problems from the data set with different problem sizes and different tightness of the capacity constraint are chosen to evaluate the performance of the SA heuristics with drop/add neighborhood structure. The probability of accepting non-improving candidate solutions are set at $80 \%$ and $90 \%$. The other parameters were fixed for both cases. That is, the number of iterations at each temperature $N(t)$ is defined to be $10 \%$ of the size of the 
neighborhood, and the cooling parameter $\alpha$ is set to 0.99 . See the results of this preliminary study in Table 5.1 below. The $90 \%$ case (i.e., $90 \%$ probability of accepting non-improving candidate solutions) gave better results. Therefore, this is used in the later experiments.

Table 5.1: Setting Initial Temperature for SA Heuristics

\begin{tabular}{|c|c|c|c|c|c|c|c|c|c|}
\hline \multirow{2}{*}{$\begin{array}{c}\text { Probability } \\
\text { of } \\
\text { accepting } \\
\text { candidate } \\
\text { solutions }\end{array}$} & \multirow{2}{*}{$\begin{array}{l}\text { Problem } \\
\text { Instances }\end{array}$} & \multirow{2}{*}{$\begin{array}{c}\text { Best } \\
\text { solution } \\
\text { in } \\
\text { literature }\end{array}$} & \multirow[t]{2}{*}{ Trial 1} & \multirow[t]{2}{*}{ Trial 2} & \multirow[t]{2}{*}{ Trial 3} & \multicolumn{2}{|c|}{ Average } & \multicolumn{2}{|c|}{ Best } \\
\hline & & & & & & Cost & $\begin{array}{c}\% \\
\text { Deviation }\end{array}$ & Cost & $\begin{array}{c}\% \\
\text { Deviation }\end{array}$ \\
\hline \multirow[t]{3}{*}{$80 \%$} & $20-15-35$ & 1471896 & 1718733 & 1781154 & 1614116 & 1704668 & $15.81 \%$ & 1614116 & $9.66 \%$ \\
\hline & $35-15-55$ & 4639128 & 4913912 & 5486242 & 5456673 & 5285609 & $13.94 \%$ & 4913912 & $5.92 \%$ \\
\hline & $50-10-95$ & 12845598 & 18171288 & 20514591 & 19860331 & 19515403 & $51.92 \%$ & 18171288 & $41.46 \%$ \\
\hline \multirow[t]{3}{*}{$90 \%$} & $20-15-35$ & 1471896 & 1521604 & 1506076 & 1535928 & 1521203 & $3.35 \%$ & 1506076 & $2.32 \%$ \\
\hline & $35-15-55$ & 4639128 & 4819067 & 4944205 & 4818008 & 4860427 & $4.77 \%$ & 4818008 & $3.86 \%$ \\
\hline & $50-10-95$ & 12845598 & 18052809 & 17049092 & 17380906 & 17494269 & $36.19 \%$ & 17049092 & $32.72 \%$ \\
\hline
\end{tabular}

\section{Minimum Allowable Temperature, $T_{\min }$}

Minimum allowable temperature is used as the stopping criterion in the proposed SA heuristics. The minimum allowable temperature, $T_{\min }$ is set to 0.01, as in McKendall et. al, 2006.

\section{Cooling Ratio, $\alpha$}

In the SA heuristics literature, the value of the cooling ratio $\alpha$ is set between 0.9-0.998. In the experiments below, this value is set to 0.99 .

4. Number of iterations at each temperature, $N(t)$

Using the parameter settings mentioned above, a preliminary experiment was performed to see how the value of $N(t)$ affects the results. The values of $N(t)$ used are $10 \%, 20 \%, 30 \%, 40 \%$ and $50 \%$ of the size of the neighborhood. The results are shown in Tables 5.2-5.6. The results equal to or better than the best found solutions in literature are given in bold font. 
Table 5.2: Results with $N(t)=10 \%$ of the size of the neighborhood

\begin{tabular}{|c|c|c|c|c|c|c|c|c|c|}
\hline $\begin{array}{l}\text { Problem } \\
\text { Instances }\end{array}$ & $\begin{array}{c}\text { Best } \\
\text { solution } \\
\text { in } \\
\text { literature }\end{array}$ & Trial 1 & Trial 2 & Trial 3 & Trial 4 & Trial 5 & Trial 6 & $\begin{array}{c}\text { Best } \\
\text { Results }\end{array}$ & $\begin{array}{c}\% \\
\text { Deviation }\end{array}$ \\
\hline $20-15-35$ & 1471896 & 1562334 & 1666277 & 1654817 & 1683947 & 1633818 & 1506076 & 1506076 & $2.32 \%$ \\
\hline $20-15-55$ & 1723638 & 1774373 & 1760779 & 1746373 & 1765301 & 1768575 & 1775879 & 1746373 & $1.32 \%$ \\
\hline $20-15-75$ & 1967315 & 2164399 & 2082325 & 2197493 & 2271365 & 2110196 & 2097599 & 2082325 & $5.85 \%$ \\
\hline $30-06-95$ & 5160920 & 7343965 & 7396391 & 7282153 & 7231103 & 7261463 & 7338332 & 7231103 & $40.11 \%$ \\
\hline $30-07-75$ & 4383923 & 4866487 & 4925550 & 5261271 & 5162753 & 5043916 & 4876711 & 4866487 & $11.01 \%$ \\
\hline $30-08-55$ & 3501695 & 3604426 & 3583662 & 3640803 & 3687505 & 3501695 & 3859019 & 3501695 & $0.00 \%$ \\
\hline $30-10-65$ & 3620959 & 4061961 & 4015158 & 3983075 & 4046989 & 4317204 & 4030877 & 3983075 & $10.00 \%$ \\
\hline $30-20-35$ & 3633644 & 3470425 & 3668551 & 3786653 & 3712153 & 3806562 & 3824489 & 3470425 & $-4.49 \%$ \\
\hline $30-20-55$ & 3599602 & 3769255 & 3811693 & 3867163 & 3852868 & 3626272 & 3847910 & 3626272 & $0.74 \%$ \\
\hline $30-20-75$ & 4050938 & 4186411 & 4428979 & 4275888 & 4050938 & 4342809 & 4403353 & 4050938 & $0.00 \%$ \\
\hline $30-20-95$ & 5726530 & 7980938 & 7780961 & 8055889 & 7942314 & 7909580 & 7780961 & 7780961 & $35.88 \%$ \\
\hline $35-15-35$ & 4456670 & 4550406 & 4560935 & 4637488 & 4602290 & 4756974 & 4630814 & 4550406 & $2.10 \%$ \\
\hline $35-15-55$ & 4639128 & 4935291 & 4780621 & 5148577 & 5005799 & 4969027 & 5049512 & 4780621 & $3.05 \%$ \\
\hline $35-15-75$ & 6301723 & 6676525 & 6623197 & 6543257 & 6571093 & 6589578 & 6606436 & 6543257 & $3.83 \%$ \\
\hline $35-15-95$ & 6670264 & 11073897 & 10502375 & 10888223 & 10527428 & 11600152 & 11534541 & 10502375 & $57.45 \%$ \\
\hline $40-07-75$ & 7405793 & 7853993 & 7533579 & 7481964 & 7675760 & 7476115 & 7570414 & 7476115 & $0.95 \%$ \\
\hline $40-09-95$ & 7667719 & 9824366 & 10769720 & 10357320 & 10763954 & 10556437 & 11343446 & 9824366 & $28.13 \%$ \\
\hline $40-10-65$ & 7265559 & 7312813 & 7265559 & 7737908 & 7265559 & 7390261 & 7325468 & 7265559 & $0.00 \%$ \\
\hline $50-10-65$ & 10513029 & 10716593 & 10768262 & 10641717 & 10624654 & 10718070 & 10942884 & 10624654 & $1.06 \%$ \\
\hline $50-10-75$ & 11217503 & 12175256 & 12398118 & 11902391 & 12195717 & 12164193 & 11939694 & 11902391 & $6.11 \%$ \\
\hline $50-10-95$ & 12845598 & 17769538 & 19658267 & 15751197 & 18582569 & 18149669 & 17617411 & 15751197 & $22.62 \%$ \\
\hline
\end{tabular}


Table 5.3: Results with $N(t)=20 \%$ of the size of the neighborhood

\begin{tabular}{|c|c|c|c|c|c|c|c|c|c|}
\hline $\begin{array}{l}\text { Problem } \\
\text { Instances }\end{array}$ & $\begin{array}{c}\text { Best } \\
\text { solution } \\
\text { in } \\
\text { literature }\end{array}$ & Trial 1 & Trial 2 & Trial 3 & Trial 4 & Trial 5 & Trial 6 & $\begin{array}{c}\text { Best } \\
\text { Results }\end{array}$ & $\begin{array}{c}\% \\
\text { Deviation }\end{array}$ \\
\hline $20-15-35$ & 1471896 & 1588987 & 1548769 & 1562145 & 1601312 & 1525718 & 1521604 & 1521604 & $3.38 \%$ \\
\hline $20-15-55$ & 1723638 & 1774373 & 1764158 & 1752972 & 1817353 & 1782379 & 1723638 & 1723638 & $0.00 \%$ \\
\hline $20-15-75$ & 1967315 & 2041918 & 2126257 & 2115180 & 2057468 & 2213512 & 2120431 & 2041918 & $3.79 \%$ \\
\hline $30-06-95$ & 5160920 & 7247483 & 7160004 & 7412682 & 7475173 & 7395715 & 7497761 & 7160004 & $38.74 \%$ \\
\hline $30-07-75$ & 4383923 & 4677043 & 5347809 & 5079456 & 4678065 & 5166234 & 4594686 & 4594686 & $4.81 \%$ \\
\hline $30-08-55$ & 3501695 & 3532566 & 3501695 & 3584958 & 3532566 & 3599746 & 3638030 & 3501695 & $0.00 \%$ \\
\hline $30-10-65$ & 3620959 & 3943414 & 3941497 & 4006612 & 3777807 & 3880017 & 3976571 & 3777807 & $4.33 \%$ \\
\hline $30-20-35$ & 3633644 & 3460504 & 3731187 & 3813073 & 3830739 & 3742375 & 3724273 & 3460504 & $-4.76 \%$ \\
\hline $30-20-55$ & 3599602 & 3726465 & 3819853 & 3622258 & 3763267 & 3770997 & 3746221 & 3622258 & $0.63 \%$ \\
\hline $30-20-75$ & 4050938 & 4239695 & 4233113 & 4301731 & 4452933 & 4210272 & 4440242 & 4210272 & $3.93 \%$ \\
\hline $30-20-95$ & 5726530 & 8034139 & 7845030 & 7919961 & 7606477 & 8080334 & 8010218 & 7606477 & $32.83 \%$ \\
\hline $35-15-35$ & 4456670 & 4620810 & 4604970 & 4538589 & 4576827 & 4694835 & 4544283 & 4538589 & $1.84 \%$ \\
\hline $35-15-55$ & 4639128 & 4819067 & 4876326 & 4818008 & 5016659 & 4954007 & 4944205 & 4818008 & $3.86 \%$ \\
\hline $35-15-75$ & 6301723 & 6585733 & 6442490 & 6445856 & 6392312 & 6413066 & 6451959 & 6392312 & $1.44 \%$ \\
\hline $35-15-95$ & 6670264 & 10626593 & 10627451 & 11146052 & 10525688 & 10968883 & 10937121 & 10525688 & $57.80 \%$ \\
\hline $40-07-75$ & 7405793 & 7504465 & 7664909 & 7595950 & 7570121 & 7559801 & 7709946 & 7504465 & $1.33 \%$ \\
\hline $40-09-95$ & 7667719 & 11306285 & 9685784 & 10502930 & 10540251 & 10942780 & 10807231 & 9685784 & $26.32 \%$ \\
\hline $40-10-65$ & 7265559 & 7321819 & 7298103 & 7385528 & 7288263 & 7302026 & 7360432 & 7288263 & $0.31 \%$ \\
\hline $50-10-65$ & 10513029 & 10779442 & 10637068 & 10749210 & 10704071 & 11054040 & 10596584 & 10596584 & $0.79 \%$ \\
\hline $50-10-75$ & 11217503 & 12012667 & 12284677 & 11821395 & 11752506 & 12055603 & 12605241 & 11752506 & $4.77 \%$ \\
\hline $50-10-95$ & 12845598 & 17439024 & 17059700 & 17530023 & 18013567 & 17043366 & 17355431 & 17043366 & $32.68 \%$ \\
\hline
\end{tabular}


Table 5.4: Results with $N(t)=30 \%$ of the size of the neighborhood

\begin{tabular}{|c|c|c|c|c|c|c|c|c|c|}
\hline $\begin{array}{l}\text { Problem } \\
\text { Instances }\end{array}$ & $\begin{array}{c}\text { Best } \\
\text { solution } \\
\text { in } \\
\text { literature }\end{array}$ & Trial 1 & Trial 2 & Trial 3 & Trial 4 & Trial 5 & Trial 6 & $\begin{array}{c}\text { Best } \\
\text { Results }\end{array}$ & $\begin{array}{c}\% \\
\text { Deviation }\end{array}$ \\
\hline $20-15-35$ & 1471896 & 1559407 & 1535928 & 1584946 & 1519070 & 1556821 & 1511402 & 1511402 & $2.68 \%$ \\
\hline $20-15-55$ & 1723638 & 1738832 & 1738832 & 1761175 & 1839191 & 1775879 & 1751669 & 1738832 & $0.88 \%$ \\
\hline $20-15-75$ & 1967315 & 2318448 & 2391599 & 2153335 & 2015529 & 2058889 & 2179943 & 2015529 & $2.45 \%$ \\
\hline $30-06-95$ & 5160920 & 7409304 & 7178809 & 7340597 & 7191420 & 7250003 & 7104924 & 7104924 & $37.67 \%$ \\
\hline $30-07-75$ & 4383923 & 5283280 & 4570820 & 5047180 & 4732311 & 4402834 & 4718853 & 4402834 & $0.43 \%$ \\
\hline $30-08-55$ & 3501695 & 3532566 & 3501695 & 3501695 & 3585428 & 3622115 & 3501695 & 3501695 & $0.00 \%$ \\
\hline $30-10-65$ & 3620959 & 4126158 & 4028574 & 3959570 & 3693834 & 3785624 & 3847868 & 3693834 & $2.01 \%$ \\
\hline $30-20-35$ & 3633644 & 3710782 & 3489490 & 3724920 & 3845483 & 3380252 & 3791180 & 3380252 & $-6.97 \%$ \\
\hline $30-20-55$ & 3599602 & 3679311 & 3717663 & 3672132 & 3664287 & 3786701 & 3794916 & 3664287 & $1.80 \%$ \\
\hline $30-20-75$ & 4050938 & 4407066 & 4331100 & 4263142 & 4152522 & 4238119 & 4336481 & 4152522 & $2.51 \%$ \\
\hline $30-20-95$ & 5726530 & 7780929 & 8300102 & 8114864 & 7999346 & 8071604 & 7908528 & 7780929 & $35.88 \%$ \\
\hline $35-15-35$ & 4456670 & 4568304 & 4623489 & 4578428 & 4475921 & 4665918 & 4551509 & 4475921 & $0.43 \%$ \\
\hline $35-15-55$ & 4639128 & 4833868 & 4765403 & 4817371 & 4833912 & 4843301 & 4843082 & 4765403 & $2.72 \%$ \\
\hline $35-15-75$ & 6301723 & 6397501 & 6496238 & 6349183 & 6380077 & 6390791 & 6350896 & 6349183 & $0.75 \%$ \\
\hline $35-15-95$ & 6670264 & 10611932 & 11166777 & 11392651 & 10630882 & 10786901 & 10603120 & 10603120 & $58.96 \%$ \\
\hline $40-07-75$ & 7405793 & 7581276 & 7508405 & 7608138 & 7530671 & 7599048 & 7592428 & 7508405 & $1.39 \%$ \\
\hline $40-09-95$ & 7667719 & 11210421 & 10736737 & 10429446 & 9355239 & 10624813 & 11254917 & 9355239 & $22.01 \%$ \\
\hline $40-10-65$ & 7265559 & 7321819 & 7344992 & 7286973 & 7405811 & 7291789 & 7286973 & 7286973 & $0.29 \%$ \\
\hline $50-10-65$ & 10513029 & 10696816 & 10513029 & 10637184 & 10656900 & 10654528 & 10715283 & 10513029 & $0.00 \%$ \\
\hline $50-10-75$ & 11217503 & 12177476 & 12018025 & 12439906 & 12413982 & 12042092 & 11967764 & 11967764 & $6.69 \%$ \\
\hline $50-10-95$ & 12845598 & 18274578 & 18422922 & 16217825 & 19025566 & 18052809 & 16129920 & 16129920 & $25.57 \%$ \\
\hline
\end{tabular}


Table 5.5: Results with $N(t)=40 \%$ of the size of the neighborhood

\begin{tabular}{|c|c|c|c|c|c|c|c|c|c|}
\hline $\begin{array}{l}\text { Problem } \\
\text { Instances }\end{array}$ & $\begin{array}{c}\text { Best } \\
\text { solution } \\
\text { in } \\
\text { literature }\end{array}$ & Trial 1 & Trial 2 & Trial 3 & Trial 4 & Trial 5 & Trial 6 & $\begin{array}{c}\text { Best } \\
\text { Results }\end{array}$ & $\begin{array}{c}\% \\
\text { Deviation }\end{array}$ \\
\hline $20-15-35$ & 1471896 & 1559884 & 1556917 & 1643634 & 1528175 & 1600962 & 1550655 & 1528175 & $3.82 \%$ \\
\hline $20-15-55$ & 1723638 & 1748942 & 1794024 & 1768575 & 1760779 & 1765301 & 1757978 & 1748942 & $1.47 \%$ \\
\hline $20-15-75$ & 1967315 & 2083803 & 2063520 & 2134400 & 2028103 & 2514987 & 2108292 & 2028103 & $3.09 \%$ \\
\hline $30-06-95$ & 5160920 & 7151151 & 7104924 & 7102726 & 7432501 & 7286580 & 7263728 & 7102726 & $37.63 \%$ \\
\hline $30-07-75$ & 4383923 & 4587834 & 4657508 & 4652654 & 4676684 & 4782071 & 4641935 & 4587834 & $4.65 \%$ \\
\hline $30-08-55$ & 3501695 & 3591805 & 3532566 & 3501695 & 3552573 & 3501695 & 3532566 & 3501695 & $0.00 \%$ \\
\hline $30-10-65$ & 3620959 & 3891258 & 3863265 & 3893366 & 3840977 & 3935145 & 3871526 & 3840977 & $6.08 \%$ \\
\hline $30-20-35$ & 3633644 & 3530892 & 3781576 & 3730253 & 3765343 & 3737191 & 3739933 & 3530892 & $-2.83 \%$ \\
\hline $30-20-55$ & 3599602 & 3764621 & 4389471 & 3930419 & 3862648 & 3745105 & 3754261 & 3745105 & $4.04 \%$ \\
\hline $30-20-75$ & 4050938 & 4133686 & 4319506 & 4504684 & 4407599 & 4130034 & 4255918 & 4130034 & $1.95 \%$ \\
\hline $30-20-95$ & 5726530 & 8251191 & 8055889 & 7871059 & 8069134 & 7909580 & 7573947 & 7573947 & $32.26 \%$ \\
\hline $35-15-35$ & 4456670 & 4498330 & 4549263 & 4511450 & 4635500 & 4475921 & 4584288 & 4475921 & $0.43 \%$ \\
\hline $35-15-55$ & 4639128 & 4854988 & 4848034 & 4749599 & 4845739 & 4959720 & 4738547 & 4738547 & $2.14 \%$ \\
\hline $35-15-75$ & 6301723 & 6392902 & 6464192 & 6559522 & 6318744 & 6403600 & 6452743 & 6318744 & $0.27 \%$ \\
\hline $35-15-95$ & 6670264 & 10861275 & 10499851 & 11314476 & 10488040 & 10298816 & 10504337 & 10298816 & $54.40 \%$ \\
\hline $40-07-75$ & 7405793 & 7663150 & 7545165 & 7639618 & 7629281 & 7537946 & 7473559 & 7473559 & $0.92 \%$ \\
\hline $40-09-95$ & 7667719 & 10821908 & 9755471 & 10391745 & 9882145 & 11111991 & 10012516 & 9755471 & $27.23 \%$ \\
\hline $40-10-65$ & 7265559 & 7321429 & 7265559 & 7298103 & 7325376 & 7265559 & 7308532 & 7265559 & $0.00 \%$ \\
\hline $50-10-65$ & 10513029 & 10672779 & 10674227 & 10614690 & 10744411 & 10513029 & 10609765 & 10513029 & $0.00 \%$ \\
\hline $50-10-75$ & 11217503 & 12149323 & 11861248 & 12262942 & 11870200 & 11626712 & 12423208 & 11626712 & $3.65 \%$ \\
\hline $50-10-95$ & 12845598 & 17584924 & 17049092 & 16681197 & 16569118 & 17014096 & 17380906 & 16569118 & $28.99 \%$ \\
\hline
\end{tabular}


Table 5.6: Results with $N(t)=50 \%$ of the size of the neighborhood

\begin{tabular}{|c|c|c|c|c|c|c|c|c|c|}
\hline $\begin{array}{l}\text { Problem } \\
\text { Instances }\end{array}$ & $\begin{array}{c}\text { Best } \\
\text { solution } \\
\text { in } \\
\text { literature }\end{array}$ & Trial 1 & Trial 2 & Trial 3 & Trial 4 & Trial 5 & Trial 6 & $\begin{array}{c}\text { Best } \\
\text { Results }\end{array}$ & $\begin{array}{c}\% \\
\text { Deviation }\end{array}$ \\
\hline $20-15-35$ & 1471896 & 1558679 & 1540827 & 1688644 & 1577173 & 1506076 & 1568709 & 1506076 & $2.32 \%$ \\
\hline $20-15-55$ & 1723638 & 1748802 & 1750956 & 1749239 & 1723638 & 1723638 & 1723638 & 1723638 & $0.00 \%$ \\
\hline $20-15-75$ & 1967315 & 2512615 & 2136576 & 2069258 & 2037924 & 2046022 & 2089578 & 2037924 & $3.59 \%$ \\
\hline $30-06-95$ & 5160920 & 7115392 & 7380273 & 7039637 & 7473350 & 6737629 & 7271187 & 6737629 & $30.55 \%$ \\
\hline $30-07-75$ & 4383923 & 4721240 & 4712002 & 5193019 & 4792308 & 4645271 & 4918105 & 4645271 & $5.96 \%$ \\
\hline $30-08-55$ & 3501695 & 3532566 & 3717046 & 3574190 & 3501695 & 3501695 & 3585269 & 3501695 & $0.00 \%$ \\
\hline $30-10-65$ & 3620959 & 4132036 & 3938842 & 3675880 & 4061888 & 3980126 & 3832514 & 3675880 & $1.52 \%$ \\
\hline $30-20-35$ & 3633644 & 3735999 & 3744394 & 3697497 & 3507855 & 3775495 & 3379359 & 3379359 & $-7.00 \%$ \\
\hline $30-20-55$ & 3599602 & 3681638 & 3739926 & 3815328 & 3794765 & 3748363 & 3726853 & 3681638 & $2.28 \%$ \\
\hline $30-20-75$ & 4050938 & 4206006 & 4275035 & 4344092 & 4208611 & 4356155 & 4445755 & 4206006 & $3.83 \%$ \\
\hline $30-20-95$ & 5726530 & 7909580 & 7780961 & 8060757 & 7703063 & 7854754 & 7919961 & 7703063 & $34.52 \%$ \\
\hline $35-15-35$ & 4456670 & 4518355 & 4488838 & 4514053 & 4494650 & 4540174 & 4466588 & 4466588 & $0.22 \%$ \\
\hline $35-15-55$ & 4639128 & 4708530 & 4840727 & 4657037 & 4860235 & 4895690 & 4863835 & 4657037 & $0.39 \%$ \\
\hline $35-15-75$ & 6301723 & 6487853 & 6458198 & 6316674 & 6475040 & 6301723 & 6401514 & 6301723 & $0.00 \%$ \\
\hline $35-15-95$ & 6670264 & 10995700 & 10152100 & 10937121 & 10657443 & 11124050 & 10600533 & 10152100 & $52.20 \%$ \\
\hline $40-07-75$ & 7405793 & 7624973 & 7592188 & 7481964 & 7508644 & 7456976 & 7520676 & 7456976 & $0.69 \%$ \\
\hline $40-09-95$ & 7667719 & 10624137 & 11771971 & 11016515 & 10954067 & 10891649 & 10275821 & 10275821 & $34.01 \%$ \\
\hline $40-10-65$ & 7265559 & 7308514 & 7288263 & 7265559 & 7305381 & 7378020 & 7325376 & 7265559 & $0.00 \%$ \\
\hline $50-10-65$ & 10513029 & 10614690 & 10617874 & 10614494 & 10513029 & 10617874 & 10616746 & 10513029 & $0.00 \%$ \\
\hline $50-10-75$ & 11217503 & 12115902 & 12302901 & 12065104 & 12139666 & 11832519 & 12469026 & 11832519 & $5.48 \%$ \\
\hline $50-10-95$ & 12845598 & 17997798 & 16139493 & 17915442 & 17107898 & 17621964 & 16884109 & 16139493 & $25.64 \%$ \\
\hline
\end{tabular}

The percent deviations obtained in the five tables above are shown in a bar chart. See Table 5.7 below. Notice that $N(t)=50 \%$ of the size of the neighborhood performed better for most of the 
problems. For example, consider the problem instance 35-15-75. The percent deviations are $3.83 \%, 1.44 \%, 0.75 \%, 0.27 \%$, and $0.00 \%$ where $N(t)=10 \%, 20 \%, 30 \%, 40 \%$ and $50 \%$ of the size of the neighborhood, respectively. But the percent deviations for the problems with high space capacity utilization are still very high. For example, for the problem instance 35-15-95, the percent deviations are ranged from $52.20 \%$ to $58.96 \%$, which are very high values.

Table 5.7: Bar Chart with Percent Deviation for Different values of $N(t)$

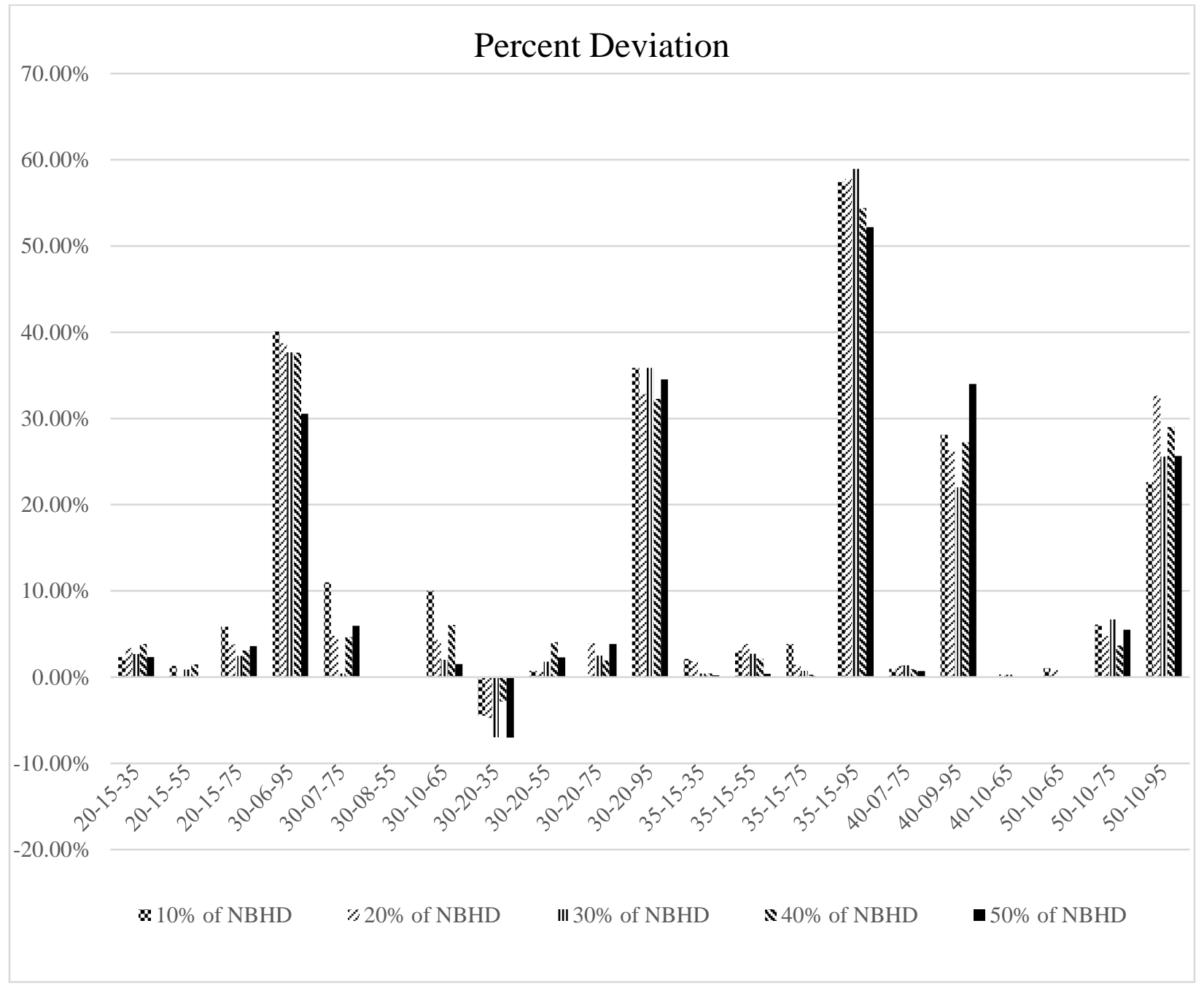

The value of the cooling ratio, $\alpha$ is now increased to 0.9999 and the minimum allowable temperature, $T_{\min }$ is lowered to 0.0001 so that we can explore more solutions and chance of getting better solutions increases. All parameters used for the proposed SA heuristics are summarized below. 
Initial temperature, $T(0)$ : the probability of accepting a non-improving neighboring solution with a cost of $10 \%$ above the cost of the initial solution is $90 \%$. See section 4.7.3.1 for the formula and explanation for calculating $T(0)$ where here $x=10$.

Cooling ratio, $\alpha=0.9999$

Number of iterations at each temperature $N(t)=50 \%$ of the size of the neighborhood

Minimum allowable temperature, $T_{\min }=0.0001$

These parameter settings are used to test the performances of the proposed SA heuristics for both neighborhood structures (i.e., drop/add and pairwise exchange). The results are presented and discussed below in Section 5.4.1.

\subsubsection{Parameters in Tabu Search (TS) Heuristics}

1. Tabu size or tabu length

For the proposed TS heuristics with both drop/add and pairwise exchange neighborhood structures, the tabu length (tabusize) is set to the exact values used in McKendall and Li (2017).

McKendall and Li (2017) obtained the best feasible solutions for the GQAP while using the tabu length by multiplying the size of the neighborhood by 0.005 to 0.02 . Smaller values of tabusize tend to result in poor solutions, since the TS heuristic either spends too much time repeating the same moves, or may get trapped at a local optima (i.e. cycling). On the other hand, using a large value for tabusize results in restricting the solution space too much whereby many potentially good moves may be overlooked, resulting in poor solutions. The proposed TS heuristics with only drop/add neighborhood structure used tabusize equal to 0.02 and 0.03 times the size of the neighborhood.

\section{Computational time}

The stopping criterion for the proposed TS heuristic is computational time. The maximum computational time (see Table 5.8 and 5.10) for each problem from SA heuristic is set as the computational time for the respective problems.

These parameter settings are used to test the performances of the proposed TS heuristics. The results are presented and discussed below, in Section 5.4.2. 


\section{$5.4 \quad$ Results}

As stated previously, the proposed TS and SA heuristics used the dataset from Cordeau et. al. (2006) to test the performances of the proposed heuristics. The results are presented below.

\subsubsection{Simulated Annealing (SA) Heuristics}

The proposed SA heuristics was implemented using the parameter settings mentioned in Section 5.3.1. The results of the SA heuristics with drop/add neighborhood structure is given in Table 5.8.

Table 5.8: Results from SA Heuristics with Drop/Add Neighborhood

\begin{tabular}{|c|c|c|c|c|c|c|c|c|c|c|c|}
\hline \multirow{2}{*}{$\begin{array}{l}\text { Problem } \\
\text { Instances }\end{array}$} & \multirow[t]{2}{*}{ Trial 1} & \multirow[t]{2}{*}{ Trial 2} & \multirow[t]{2}{*}{ Trial 3} & \multirow[t]{2}{*}{ Trial 4} & \multirow[t]{2}{*}{ Trial 5} & \multicolumn{2}{|c|}{ Average } & \multicolumn{2}{|c|}{ Best } & \multirow{2}{*}{$\begin{array}{l}\text { Average } \\
\text { Runtime } \\
\text { (seconds) }\end{array}$} & \multirow{2}{*}{$\begin{array}{c}\text { Maximum } \\
\text { Runtime } \\
\text { (seconds) }\end{array}$} \\
\hline & & & & & & Cost & $\begin{array}{c}\% \\
\text { Deviation }\end{array}$ & Cost & $\begin{array}{c}\% \% \\
\text { Deviation }\end{array}$ & & \\
\hline $20-15-35$ & 1506076 & 1539678 & 1509123 & 1511402 & 1511402 & 1515536.2 & $2.96 \%$ & 1506076 & $2.32 \%$ & 3831.8 & 4194.7 \\
\hline $20-15-55$ & 1723638 & 1723638 & 1723638 & 1723638 & 1723638 & 1723638 & $0.00 \%$ & \begin{tabular}{|l|l|}
1723638 \\
\end{tabular} & $0.00 \%$ & 4092.7 & 4457.5 \\
\hline $20-15-75$ & 1987133 & 1988689 & 1988689 & 1976791 & 1989271 & 1986114.6 & $0.96 \%$ & 1976791 & $0.48 \%$ & 4266.9 & 4565.4 \\
\hline $30-06-95$ & 6416228 & 6219342 & 6476590 & 6513339 & 6416329 & 6408365.6 & $24.17 \%$ & 6219342 & $20.51 \%$ & 1154.6 & 1212.5 \\
\hline $30-07-75$ & 4540060 & 4640070 & 4600906 & 4595501 & 4400593 & 4555426 & $3.91 \%$ & 4400593 & $0.38 \%$ & 2047.1 & 2287.2 \\
\hline $30-08-55$ & 3501695 & 3501695 & 3501695 & 3501695 & 3501695 & 3501695 & $0.00 \%$ & 3501695 & $0.00 \%$ & 2707.6 & 2962.6 \\
\hline $30-10-65$ & 3663198 & 3746644 & 3736251 & 3640684 & 3713822 & 3700119.8 & $2.19 \%$ & 3640684 & $0.54 \%$ & 3557.4 & 3767.8 \\
\hline $30-20-35$ & 3510015 & 3380252 & 3451254 & 3380252 & 3468225 & 3437999.6 & $-5.38 \%$ & 3380252 & $-6.97 \%$ & 8924.0 & 9672.0 \\
\hline $30-20-55$ & 3593105 & 3626509 & 3622258 & 3649512 & 3660919 & 3630460.6 & $0.86 \%$ & 3593105 & $-0.18 \%$ & 9414.6 & 10907.9 \\
\hline $30-20-75$ & 4050938 & 4116011 & 4215750 & 4115879 & 4107614 & 4121238.4 & $1.74 \%$ & 4050938 & $0.00 \%$ & 10405.2 & 11363.0 \\
\hline $30-20-95$ & 7573110 & 7575493 & 7573110 & 7575493 & 7573110 & 7574063.2 & $32.26 \%$ & 7573110 & $32.25 \%$ & 16476.4 & 17004.0 \\
\hline $35-15-35$ & 4456670 & 4457348 & 4459912 & 4464070 & 4456670 & 4458934 & $0.05 \%$ & 4456670 & $0.00 \%$ & 7024.2 & 7714.4 \\
\hline $35-15-55$ & 4713054 & 4722172 & 4673657 & 4639128 & 4670370 & 4683676.2 & $0.96 \%$ & 4639128 & $0.00 \%$ & 7436.5 & 7974.1 \\
\hline $35-15-75$ & 6319838 & 6319838 & 6319838 & 6301723 & 6332985 & 6318844.4 & $0.27 \%$ & 6301723 & $0.00 \%$ & 7846.6 & 8936.8 \\
\hline $35-15-95$ & 9089146 & 9917835 & 9089146 & 10108923 & 10000498 & 9641109.6 & $44.54 \%$ & 9089146 & $36.26 \%$ & 8632.8 & 10332.0 \\
\hline $40-07-75$ & 7466532 & 7458588 & 7478567 & 7469028 & 7428176 & 7460178.2 & $0.73 \%$ & 7428176 & $0.30 \%$ & 2797.8 & 2956.6 \\
\hline 40-09-95 & 9189719 & 9619006 & 9985473 & 9499848 & 9546826 & 9568174.4 & $24.79 \%$ & 9189719 & $19.85 \%$ & 3658.7 & 3915.8 \\
\hline $40-10-65$ & 7265559 & 7286973 & 7274192 & 7285016 & 7265559 & 7275459.8 & $0.14 \%$ & 7265559 & $0.00 \%$ & 4976.9 & 5214.0 \\
\hline $50-10-65$ & 10513029 & 10513029 & 10513029 & 10513029 & 10513029 & 10513029 & $0.00 \%$ & 10513029 & $0.00 \%$ & 6312.7 & 6713.1 \\
\hline 50-10-75 & 11967764 & 11935565 & 12096401 & 11482321 & 11637986 & 11824007 & $5.41 \%$ & 11482321 & $2.36 \%$ & 6032.5 & 6340.1 \\
\hline 50-10-95 & 14278379 & 15335045 & 14964326 & 14115461 & 14455373 & 14629717 & $13.89 \%$ & 14115461 & $9.89 \%$ & 3831.8 & 6058.7 \\
\hline
\end{tabular}


Table 5.9 gives a bar chart of the results presented in Tables $5.7 \& 5.8$ (i.e., with a new column which represents the percent deviations with the new parameter settings (see "Final Results")). For example, consider problem instance 30-10-65. With the previous parameter setting the best result was $1.52 \%$ deviation (see Table 5.6). With the new parameter setting $0.54 \%$ deviation (see Table 5.8 ) is obtained. Consider another problem instance 35-15-55. With the previous parameter setting the best result was $0.39 \%$ deviation (see Table 5.6). With the new parameter setting $0.0 \%$ deviation is obtained i.e. the solution is equal to the best solution found in literature. Also, notice that the deviations for the problems with high space capacity utilization have decreased and it produced better results for other problems too. For example, the problem instance 50-10-95 is a problem with high space capacity utilization of 95 . With the previous parameter setting the best result was $22.62 \%$ deviation (see Table 5.2). With the new parameter setting 9.89\% deviation (see Table 5.8) is obtained.

\section{Table 5.9: Bar Chart with Percent Deviations}

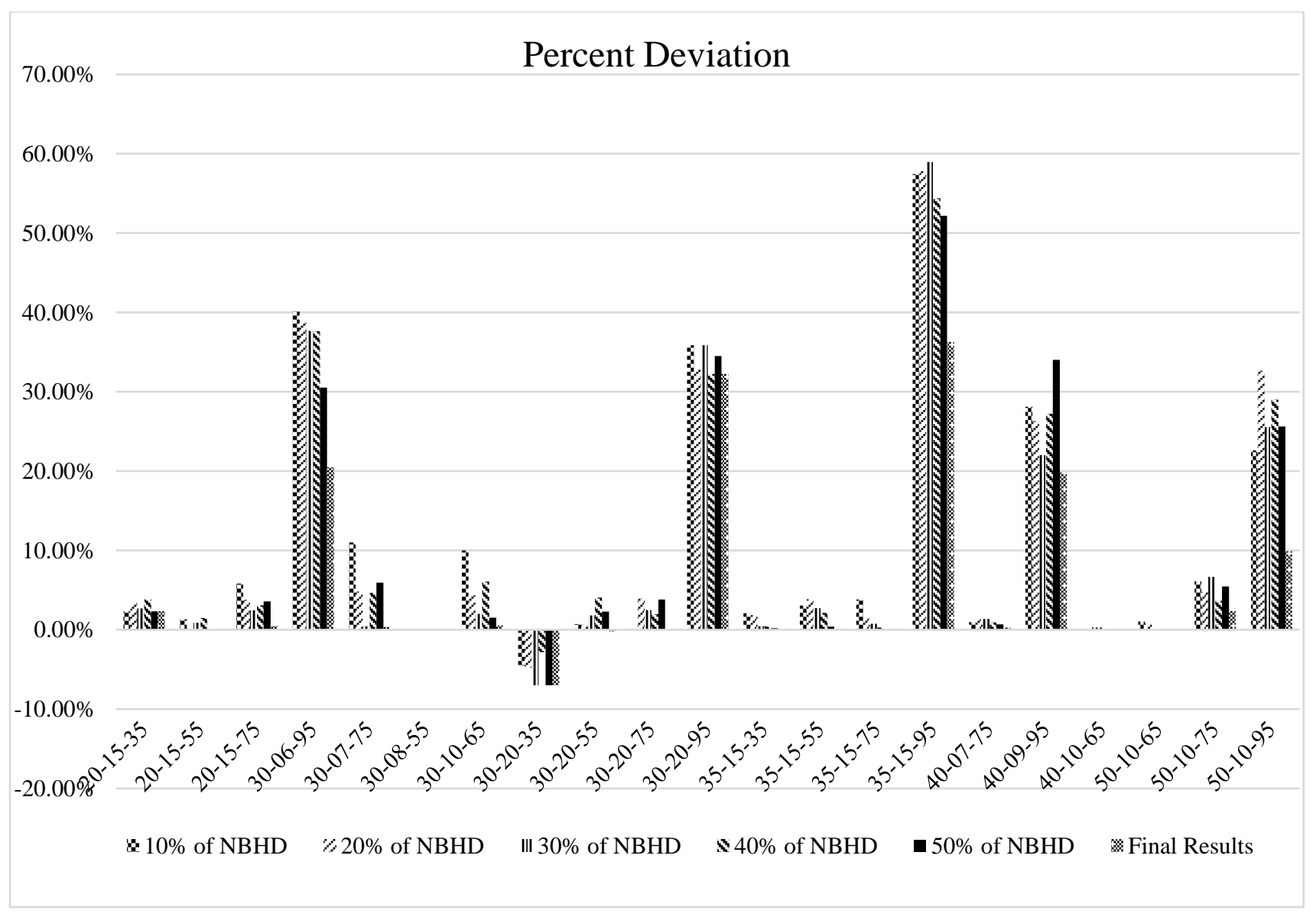


The same values of the parameter settings are used for the SA with both neighborhood structures. The results are shown in Table 5.10. Results for three (3) test problems are better than the results presented in literature. The three problem instances are 20-15-75, 30-20-35 and 30-20-55. The percent reductions are $-0.72 \%,-7.00 \%$, and $-0.18 \%$, respectively (i.e., the costs for these problems are $0.72 \%, 7.00 \%$, and $0.18 \%$ less than the best found solution for the above mentioned problems). Also, 14 results are exactly equal to the best results found in literature. The other 4 results are worse than the best found results, but the percent deviations for these problems are less than $1 \%$ (i.e., the costs are less than $1 \%$ above the best found results).

Table 5.10: Results from SA Heuristics with Both Neighborhoods

\begin{tabular}{|c|c|c|c|c|c|c|c|c|c|c|c|}
\hline \multirow{2}{*}{$\begin{array}{l}\text { Problem } \\
\text { Instances }\end{array}$} & \multirow[t]{2}{*}{ Trial 1} & \multirow[t]{2}{*}{ Trial 2} & \multirow[t]{2}{*}{ Trial 3} & \multirow[t]{2}{*}{ Trial 4} & \multirow[t]{2}{*}{ Trial 5} & \multicolumn{2}{|c|}{ Average } & \multicolumn{2}{|c|}{ Best } & \multirow{2}{*}{$\begin{array}{l}\text { Average } \\
\text { Runtime } \\
\text { (seconds) }\end{array}$} & \multirow{2}{*}{$\begin{array}{c}\text { Maximum } \\
\text { Runtime } \\
\text { (seconds) }\end{array}$} \\
\hline & & & & & & Cost & $\begin{array}{c}\% \\
\text { Deviation }\end{array}$ & Cost & $\begin{array}{c}\% \\
\text { Deviation }\end{array}$ & & \\
\hline $20-15-35$ & 1471896 & 1471896 & 1471896 & 1471896 & 1471896 & 1471896 & $0.00 \%$ & 1471896 & $0.00 \%$ & 4161.6 & 4289.1 \\
\hline $20-15-55$ & 1723638 & 1723638 & 1723638 & 1723638 & 1723638 & 1723638 & $0.00 \%$ & 1723638 & $0.00 \%$ & 4470.4 & 4680.3 \\
\hline $20-15-75$ & 1953188 & 1953188 & 1953188 & 1953188 & 1953188 & 1953188 & $-0.72 \%$ & 1953188 & $-0.72 \%$ & 4494.3 & 4595.7 \\
\hline $30-06-95$ & 5160920 & 5160920 & 5160920 & 5160920 & 5160920 & 5160920 & $0.00 \%$ & 5160920 & $0.00 \%$ & 1576.3 & 1597.8 \\
\hline $30-07-75$ & 4383923 & 4383923 & 4383923 & 4383923 & 4383923 & 4383923 & $0.00 \%$ & 4383923 & $0.00 \%$ & 2238.1 & 2406.7 \\
\hline $30-08-55$ & 3501695 & 3501695 & 3501695 & 3501695 & 3501695 & 3501695 & $0.00 \%$ & 3501695 & $0.00 \%$ & 2771.6 & 2969.4 \\
\hline $30-10-65$ & 3654908 & 3620959 & 3620959 & 3620959 & 3620959 & 3627748.8 & $0.19 \%$ & 3620959 & $0.00 \%$ & 3758.9 & 3957.7 \\
\hline $30-20-35$ & 3379359 & 3379359 & 3379359 & 3379359 & 3379359 & 3379359 & $-7.00 \%$ & 3379359 & $-7.00 \%$ & 9835.7 & 10190.0 \\
\hline $30-20-55$ & 3593105 & 3593105 & 3593105 & 3593105 & 3593105 & 3593105 & $-0.18 \%$ & 3593105 & $-0.18 \%$ & 10460.1 & 10910.0 \\
\hline $30-20-75$ & 4050938 & 4050938 & 4050938 & 4104358 & 4104358 & 4072306 & $0.53 \%$ & 4050938 & $0.00 \%$ & 11526.5 & 12212.5 \\
\hline $30-20-95$ & 5759808 & 5961222 & 5804873 & 5961222 & 5759808 & 5849386.6 & $2.15 \%$ & 5759808 & $0.58 \%$ & 16138.3 & 16631.2 \\
\hline $35-15-35$ & 4457348 & 4457348 & 4456670 & 4457348 & 4457348 & 4457212.4 & $0.01 \%$ & 4456670 & $0.00 \%$ & 7720.9 & 7963.4 \\
\hline $35-15-55$ & 4639128 & 4639128 & 4639128 & 4639128 & 4639128 & 4639128 & $0.00 \%$ & 4639128 & $0.00 \%$ & 8053.0 & 8371.3 \\
\hline $35-15-75$ & 6319838 & 6316674 & 6301723 & 6316674 & 6316674 & 6314316.6 & $0.20 \%$ & 6301723 & $0.00 \%$ & 9176.7 & 11094.6 \\
\hline $35-15-95$ & 6811310 & 6777590 & 6713937 & 6751402 & 6751402 & 6761128.2 & $1.36 \%$ & 6713937 & $0.65 \%$ & 10005.3 & 11005.5 \\
\hline $40-07-75$ & 7405793 & 7432555 & 7405793 & 7432555 & 7432555 & 7421850.2 & $0.22 \%$ & 7405793 & $0.00 \%$ & 3075.8 & 3180.7 \\
\hline 40-09-95 & 7732515 & 7783476 & 7769026 & 7773594 & 7773594 & 7766441 & $1.29 \%$ & 7732515 & $0.85 \%$ & 4274.2 & 4625.3 \\
\hline $40-10-65$ & 7265559 & 7265559 & 7265559 & 7265559 & 7265559 & 7265559 & $0.00 \%$ & 7265559 & $0.00 \%$ & 5520.4 & 5765.2 \\
\hline $50-10-65$ & 10513029 & 10513029 & 10513029 & 10513029 & 10513029 & 10513029 & $0.00 \%$ & 10513029 & $0.00 \%$ & 7105.5 & 7296.1 \\
\hline $50-10-75$ & 11251072 & 11275061 & 11259251 & 11275061 & 11251072 & 11262303.4 & $0.40 \%$ & 11251072 & $0.30 \%$ & 6774.2 & 6928.5 \\
\hline 50-10-95 & 12864406 & 12845598 & 12845598 & 12845598 & 12845598 & 12849359.6 & $0.03 \%$ & 12845598 & $0.00 \%$ & 6607.8 & 6921.1 \\
\hline
\end{tabular}




\subsubsection{Tabu Search (TS) Heuristics}

Tabu Search (TS) heuristics with drop/add neighborhood structure is considered with two different set of tabu sizes (mentioned in Section 5.3.2). Tables 5.11 a and 5.11 b present the results with the tabusize equal to the size of the neighborhood (NBHD) times 0.02 and $0.03(0.02 N B H D$ and $0.03 N B H D$ ), respectively. The computation time is equal to the maximum runtime we obtained in SA heuristics with drop/add neighborhood structure. See Table 5.8 for computation times.

Table 5.11a: Results from TS Heuristics with Drop/Add Neighborhood (tabusize $=0.02 N B H D$ )

\begin{tabular}{|c|c|c|c|c|c|}
\hline $\begin{array}{c}\text { Problem } \\
\text { Instances }\end{array}$ & $\begin{array}{c}\text { Best solution } \\
\text { in literature }\end{array}$ & Cost & \% Deviation & Tabu size & $\begin{array}{c}\text { Runtime } \\
\text { (seconds) }\end{array}$ \\
\hline $20-15-35$ & 1471896 & 1555456 & $5.68 \%$ & 6 & 4194.7 \\
\hline $20-15-55$ & 1723638 & 1761175 & $2.18 \%$ & 6 & 4457.5 \\
\hline $20-15-75$ & 1967315 & 2635767 & $33.98 \%$ & 6 & 4565.4 \\
\hline $30-06-95$ & 5160920 & 7311111 & $41.66 \%$ & 3 & 1212.5 \\
\hline $30-07-75$ & 4383923 & 4964696 & $13.25 \%$ & 4 & 2287.2 \\
\hline $30-08-55$ & 3501695 & 3628917 & $3.63 \%$ & 4 & 2962.6 \\
\hline $30-10-65$ & 3620959 & 4264490 & $17.77 \%$ & 5 & 3767.8 \\
\hline $30-20-35$ & 3633644 & 3753614 & $3.30 \%$ & 11 & 9672.0 \\
\hline $30-20-55$ & 3599602 & 4067925 & $13.01 \%$ & 11 & 10907.9 \\
\hline $30-20-75$ & 4050938 & 4759033 & $17.48 \%$ & 11 & 11363.0 \\
\hline $30-20-95$ & 5726530 & 8197698 & $43.15 \%$ & 11 & 17004.0 \\
\hline $35-15-35$ & 4456670 & 4710133 & $5.69 \%$ & 10 & 7714.4 \\
\hline $35-15-55$ & 4639128 & 5046883 & $8.79 \%$ & 10 & 7974.1 \\
\hline $35-15-75$ & 6301723 & 6772916 & $7.48 \%$ & 10 & 8936.8 \\
\hline $35-15-95$ & 6670264 & 10638463 & $59.49 \%$ & 10 & 10332.0 \\
\hline $40-07-75$ & 7405793 & 8930083 & $20.58 \%$ & 5 & 2956.6 \\
\hline $40-09-95$ & 7667719 & 11240530 & $46.60 \%$ & 6 & 3915.8 \\
\hline $40-10-65$ & 7265559 & 7343987 & $1.08 \%$ & 7 & 5214.0 \\
\hline $50-10-65$ & 10513029 & 11025649 & $4.88 \%$ & 9 & 6713.1 \\
\hline $50-10-75$ & 11217503 & 13209132 & $17.75 \%$ & 9 & 6340.1 \\
\hline $50-10-95$ & 12845598 & 15962204 & $24.26 \%$ & 9 & 6058.7 \\
\hline & & & & & \\
\hline
\end{tabular}


Table 5.11b: Results from TS Heuristics with Drop/Add Neighborhood (tabusize =0.03NBHD)

\begin{tabular}{|c|c|c|c|c|c|}
\hline $\begin{array}{c}\text { Problem } \\
\text { Instances }\end{array}$ & $\begin{array}{c}\text { Best solution } \\
\text { in literature }\end{array}$ & Cost & $\%$ Deviation & Tabu size & $\begin{array}{c}\text { Runtime } \\
\text { (seconds) }\end{array}$ \\
\hline $20-15-35$ & 1471896 & 1555456 & $5.68 \%$ & 8 & 4194.7 \\
\hline $20-15-55$ & 1723638 & 1761175 & $2.18 \%$ & 8 & 4457.5 \\
\hline $20-15-75$ & 1967315 & 2635767 & $33.98 \%$ & 8 & 4565.4 \\
\hline $30-06-95$ & 5160920 & 7164409 & $38.82 \%$ & 5 & 1212.5 \\
\hline $30-07-75$ & 4383923 & 4964696 & $13.25 \%$ & 5 & 2287.2 \\
\hline $30-08-55$ & 3501695 & 3585428 & $2.39 \%$ & 6 & 2962.6 \\
\hline $30-10-65$ & 3620959 & 4264490 & $17.77 \%$ & 8 & 3767.8 \\
\hline $30-20-35$ & 3633644 & 3768876 & $3.72 \%$ & 17 & 9672.0 \\
\hline $30-20-55$ & 3599602 & 3834865 & $6.54 \%$ & 17 & 10907.9 \\
\hline $30-20-75$ & 4050938 & 4756691 & $17.42 \%$ & 17 & 11363.0 \\
\hline $30-20-95$ & 5726530 & 8197698 & $43.15 \%$ & 17 & 17004.0 \\
\hline $35-15-35$ & 4456670 & 4710133 & $5.69 \%$ & 15 & 7714.4 \\
\hline $35-15-55$ & 4639128 & 5046883 & $8.79 \%$ & 15 & 7974.1 \\
\hline $35-15-75$ & 6301723 & 6772916 & $7.48 \%$ & 15 & 8936.8 \\
\hline $35-15-95$ & 6670264 & 10638463 & $59.49 \%$ & 15 & 10332.0 \\
\hline $40-07-75$ & 7405793 & 8250469 & $11.41 \%$ & 7 & 2956.6 \\
\hline $40-09-95$ & 7667719 & 11240530 & $46.60 \%$ & 10 & 3915.8 \\
\hline $40-10-65$ & 7265559 & 7343987 & $1.08 \%$ & 11 & 5214.0 \\
\hline $50-10-65$ & 10513029 & 10921687 & $3.89 \%$ & 14 & 6713.1 \\
\hline $50-10-75$ & 11217503 & 13136023 & $17.10 \%$ & 14 & 6340.1 \\
\hline $50-10-95$ & 12845598 & 15962204 & $24.26 \%$ & 14 & 6058.7 \\
\hline
\end{tabular}

If we compare the solutions obtained from these two different tabu sizes, we can see Table 5.11b produced either same or better results compared to Table 5.11a. It can be seen more clearly in the bar chart presented in Table 5.12. 
Table 5.12: Results Obtained from TS Heuristics with Drop/Add where tabusize $=0.02 N B H D$ or $0.03 N B H D$

\section{Costs}

\section{0}

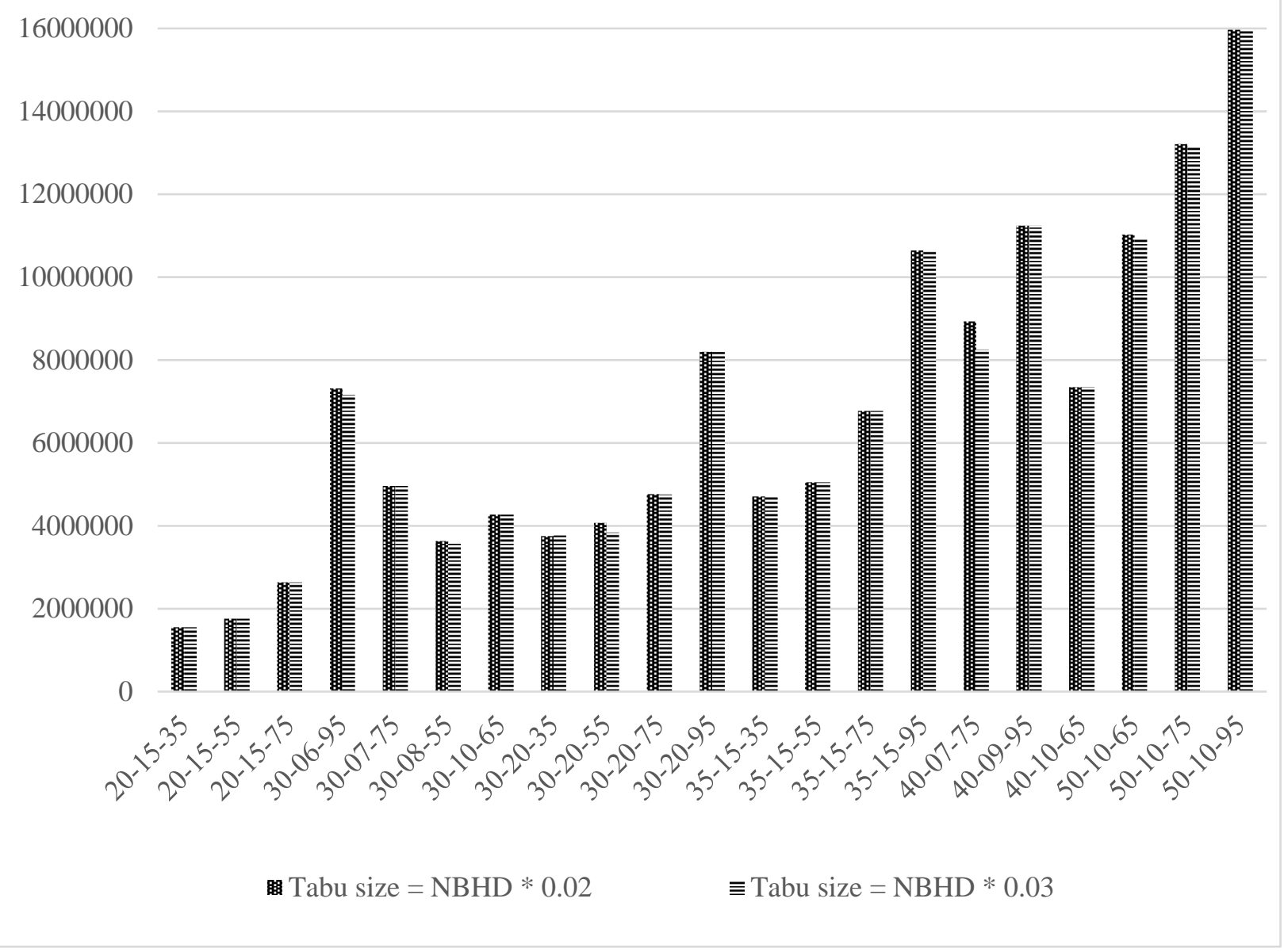

Results obtained from the TS heuristics with both drop/add and pairwise exchange neighborhood structures are given in Table 5.13. It obtained two results equal to the best found solution in literature. The computation time is equal to the maximum runtime we obtained in SA heuristics with both neighborhood structures. See Table 5.10 for computation times. 
Table 5.13: Results from TS Heuristics with Both Neighborhoods

\begin{tabular}{|c|c|c|c|c|c|}
\hline $\begin{array}{l}\text { Problem } \\
\text { Instances }\end{array}$ & $\begin{array}{c}\text { Best solution } \\
\text { in literature }\end{array}$ & Cost & $\begin{array}{c}\% \\
\text { Deviation }\end{array}$ & Tabu size & $\begin{array}{l}\text { Runtime } \\
\text { (seconds) }\end{array}$ \\
\hline $20-15-35$ & 1471896 & 1500497 & $1.94 \%$ & 8 & 4289.1 \\
\hline $20-15-55$ & 1723638 & 1723638 & $0.00 \%$ & 8 & 4680.3 \\
\hline $20-15-75$ & 1967315 & 2155374 & $9.56 \%$ & 7 & 4595.7 \\
\hline $30-06-95$ & 5160920 & 5222897 & $1.20 \%$ & 7 & 1597.8 \\
\hline $30-07-75$ & 4383923 & 4519174 & $3.09 \%$ & 7 & 2406.7 \\
\hline $30-08-55$ & 3501695 & 3578548 & $2.19 \%$ & 7 & 2969.4 \\
\hline $30-10-65$ & 3620959 & 3867245 & $6.80 \%$ & 11 & 3957.7 \\
\hline $30-20-35$ & 3633644 & 3853792 & $6.06 \%$ & 12 & 10190.0 \\
\hline $30-20-55$ & 3599602 & 3798453 & $5.52 \%$ & 12 & 10910.0 \\
\hline $30-20-75$ & 4050938 & 4417311 & $9.04 \%$ & 11 & 12212.5 \\
\hline $30-20-95$ & 5726530 & 6436452 & $12.40 \%$ & 12 & 16631.2 \\
\hline $35-15-35$ & 4456670 & 4543691 & $1.95 \%$ & 12 & 7963.4 \\
\hline $35-15-55$ & 4639128 & 4793722 & $3.33 \%$ & 10 & 8371.3 \\
\hline $35-15-75$ & 6301723 & 6432304 & $2.07 \%$ & 11 & 11094.6 \\
\hline $35-15-95$ & 6670264 & 7387460 & $10.75 \%$ & 12 & 11005.5 \\
\hline $40-07-75$ & 7405793 & 7405793 & $0.00 \%$ & 11 & 3180.7 \\
\hline $40-09-95$ & 7667719 & 8238651 & $7.45 \%$ & 8 & 4625.3 \\
\hline $40-10-65$ & 7265559 & 7308532 & $0.59 \%$ & 10 & 5765.2 \\
\hline $50-10-65$ & 10513029 & 10588213 & $0.72 \%$ & 10 & 7296.1 \\
\hline $50-10-75$ & 11217503 & 11783796 & $5.05 \%$ & 8 & 6928.5 \\
\hline $50-10-95$ & 12845598 & 13922455 & $8.38 \%$ & 10 & 6921.1 \\
\hline
\end{tabular}

\subsection{Comparison of Performances}

The results obtained from the proposed heuristics are summarized in Table 5.14. Consider the bolded results (best solutions found), notice that the SA heuristic with both drop/add and pairwise exchange neighborhood structures performed better compared to the other ones. 
Overall, when comparing the objective function values (or Cost), the proposed SA heuristics performed better than the proposed TS heuristics (see Table 5.15). Both TS and SA heuristics with only drop/add neighborhood performed poorly for the problems with high space capacity utilization. The percent deviations are very high for these problems (see Table 5.15). For example, consider the problem instance 30-06-95. The percent deviations while using both drop/add and pairwise exchange neighborhood are $0.00 \%$ (SA) and $1.20 \%$ (TS) whereas these deviations while using only drop/add neighborhood are $20.51 \%$ (SA) and $38.82 \%$ (TS).

Table 5.14: Summary of the Results

\begin{tabular}{|c|c|c|c|c|c|c|c|c|}
\hline \multirow[t]{2}{*}{$\begin{array}{l}\text { Problem } \\
\text { Instances }\end{array}$} & \multicolumn{2}{|c|}{ SA (Drop/Add) } & \multicolumn{2}{|c|}{$\begin{array}{c}\text { SA (Both } \\
\text { Neighborhood) }\end{array}$} & \multicolumn{2}{|c|}{ TS (Drop/Add) } & \multicolumn{2}{|c|}{$\begin{array}{c}\text { TS (Both } \\
\text { Neighborhood) }\end{array}$} \\
\hline & Cost & $\begin{array}{c}\% \\
\text { Deviation }\end{array}$ & Cost & $\begin{array}{c}\% \\
\text { Deviation }\end{array}$ & Cost & $\begin{array}{c}\% \\
\text { Deviation }\end{array}$ & Cost & $\begin{array}{c}\% \\
\text { Deviation }\end{array}$ \\
\hline $20-15-35$ & 1506076 & $2.32 \%$ & 1471896 & $0.00 \%$ & 1555456 & $5.68 \%$ & 1500497 & $1.94 \%$ \\
\hline $20-15-55$ & 1723638 & $0.00 \%$ & 1723638 & $0.00 \%$ & 1761175 & $2.18 \%$ & 1723638 & $0.00 \%$ \\
\hline $20-15-75$ & 1976791 & $0.48 \%$ & 1953188 & $-0.72 \%$ & 2635767 & $33.98 \%$ & 2155374 & $9.56 \%$ \\
\hline $30-06-95$ & 6219342 & $20.51 \%$ & 5160920 & $0.00 \%$ & 7164409 & $38.82 \%$ & 5222897 & $1.20 \%$ \\
\hline $30-07-75$ & 4400593 & $0.38 \%$ & 4383923 & $0.00 \%$ & 4964696 & $13.25 \%$ & 4519174 & $3.09 \%$ \\
\hline $30-08-55$ & 3501695 & $0.00 \%$ & 3501695 & $0.00 \%$ & 3585428 & $2.39 \%$ & 3578548 & $2.19 \%$ \\
\hline $30-10-65$ & 3640684 & $0.54 \%$ & 3620959 & $0.00 \%$ & 4264490 & $17.77 \%$ & 3867245 & $6.80 \%$ \\
\hline $30-20-35$ & 3380252 & $-6.97 \%$ & 3379359 & $-7.00 \%$ & 3768876 & $3.72 \%$ & 3853792 & $6.06 \%$ \\
\hline $30-20-55$ & 3593105 & $-0.18 \%$ & 3593105 & $-0.18 \%$ & 3834865 & $6.54 \%$ & 3798453 & $5.52 \%$ \\
\hline $30-20-75$ & 4050938 & $0.00 \%$ & 4050938 & $0.00 \%$ & 4756691 & $17.42 \%$ & 4417311 & $9.04 \%$ \\
\hline $30-20-95$ & 7573110 & $32.25 \%$ & 5759808 & $0.58 \%$ & 8197698 & $43.15 \%$ & 6436452 & $12.40 \%$ \\
\hline $35-15-35$ & 4456670 & $0.00 \%$ & 4456670 & $0.00 \%$ & 4710133 & $5.69 \%$ & 4543691 & $1.95 \%$ \\
\hline $35-15-55$ & 4639128 & $0.00 \%$ & 4639128 & $0.00 \%$ & 5046883 & $8.79 \%$ & 4793722 & $3.33 \%$ \\
\hline $35-15-75$ & 6301723 & $0.00 \%$ & 6301723 & $0.00 \%$ & 6772916 & $7.48 \%$ & 6432304 & $2.07 \%$ \\
\hline $35-15-95$ & 9089146 & $36.26 \%$ & 6713937 & $0.65 \%$ & 10638463 & $59.49 \%$ & 7387460 & $10.75 \%$ \\
\hline $40-07-75$ & 7428176 & $0.30 \%$ & 7405793 & $0.00 \%$ & 8250469 & $11.41 \%$ & 7405793 & $0.00 \%$ \\
\hline $40-09-95$ & 9189719 & $19.85 \%$ & 7732515 & $0.85 \%$ & 11240530 & $46.60 \%$ & 8238651 & $7.45 \%$ \\
\hline $40-10-65$ & 7265559 & $0.00 \%$ & 7265559 & $0.00 \%$ & 7343987 & $1.08 \%$ & 7308532 & $0.59 \%$ \\
\hline $50-10-65$ & 10513029 & $0.00 \%$ & 10513029 & $0.00 \%$ & 10921687 & $3.89 \%$ & 10588213 & $0.72 \%$ \\
\hline $50-10-75$ & 11482321 & $2.36 \%$ & 11251072 & $0.30 \%$ & 13136023 & $17.10 \%$ & 11783796 & $5.05 \%$ \\
\hline $50-10-95$ & 14115461 & $9.89 \%$ & 12845598 & $0.00 \%$ & 15962204 & $24.26 \%$ & 13922455 & $8.38 \%$ \\
\hline
\end{tabular}


Table 5.15: Comparison of Results for the Proposed Heuristics

\section{Total Costs}

18000000

16000000

14000000

12000000

10000000

8000000

6000000

4000000

2000000

0

Nin

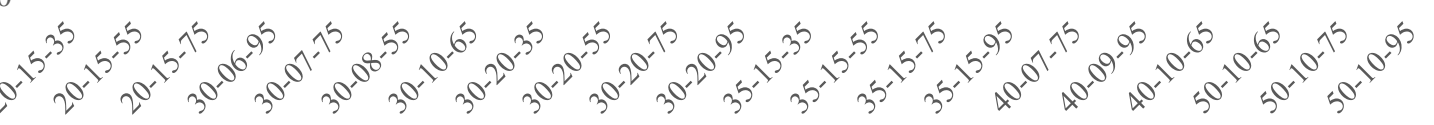

- Literature $\quad \mathbf{s}$ SA (Drop/Add) $\quad$ s SA (Both Neighborhood) $\quad$ nTS (Drop/Add) III TS (Both Neighborhood)

Table 5.16: Comparison of Percent Deviations for the Proposed Heuristics

\section{Percent Deviation}

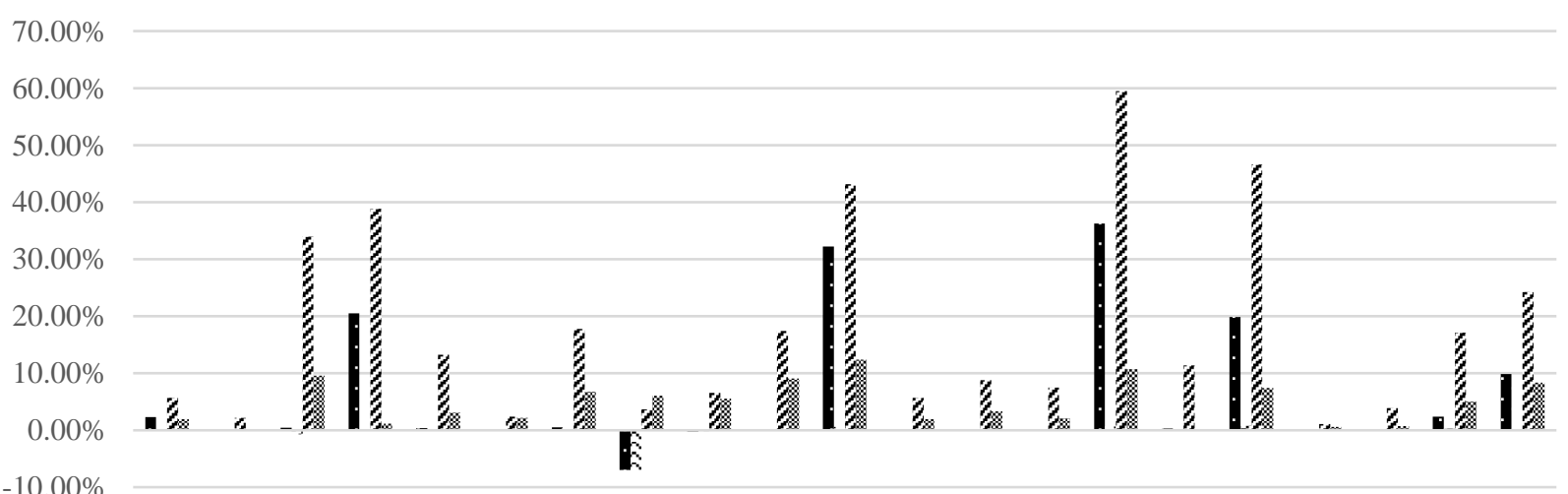

$-10.00 \%$

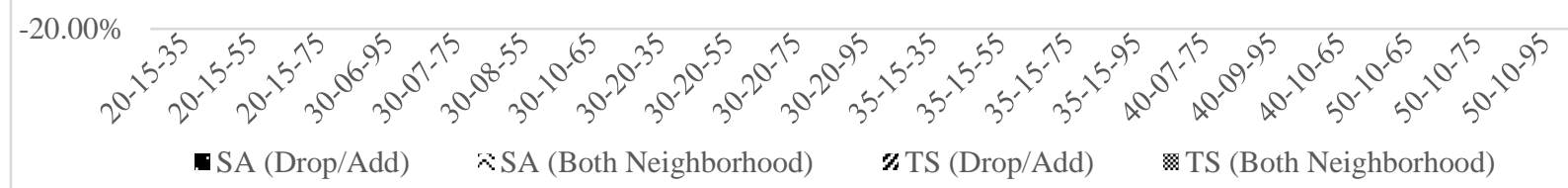


In summary, the proposed SA heuristic with both drop/add and pairwise exchange neighborhood structures outperformed the other proposed heuristics with respect to solution quality. However, the SA heuristics were run five (5) times and the maximum computation time among the five runs were used as a stopping criterion for the TS heuristic. As a result, SA heuristics were run for roughly five times the computation time of the TS heuristics. It is believed that the TS heuristics did not perform well because of it being completely deterministic. Unlike in McKendall and Li (2017) the authors added diversification to their TS heuristic by generating a set of diverse initial solutions. 


\section{CHAPTER 6 \\ CONCLUSION}

\subsection{Summary of Research}

In this research, the generalized quadratic assignment problem (GQAP) is considered and a simulated annealing (SA) and a tabu search (TS) heuristic are presented to solve the GQAP. Two types of neighborhood structures are considered for each heuristic. Either a drop/add or drop/add with pairwise exchange neighborhood structure is used.

The proposed meta-heuristics are tested on a set of 21 test problems given by Cordeau et. al. (2006). The proposed SA heuristics performed better than the proposed TS heuristics. The SA heuristics obtained results better than those presented in the literature for three of the test problems. On the other hand, the TS heuristics did not perform well for the problems with high space capacity utilization. Overall, the proposed SA heuristics performed better than the proposed TS heuristics with respect to the solution quality. As stated previously, it is believed that the proposed TS heuristics did not perform well because of it being completely deterministic. Unlike in McKendall and $\mathrm{Li} \mathrm{(2017)} \mathrm{the} \mathrm{authors} \mathrm{added} \mathrm{diversification} \mathrm{to} \mathrm{their} \mathrm{TS} \mathrm{heuristic} \mathrm{by} \mathrm{generating} \mathrm{a} \mathrm{set} \mathrm{of} \mathrm{diverse}$ initial solutions.

\subsection{Recommendations for Future Research}

The following recommendations are given for future research.

1. Consider the material handling costs between the machines assigned to the same location.

2. Consider solving the GQAP by using a hybrid technique that combines deterministic and stochastic heuristics (e.g. TS and Genetic Algorithm or TS and SA) to obtain better results.

3. Consider developing different construction algorithms to construct good initial solutions. 


\section{REFERENCES}

Abdinnour-Helm, S. and Hadley, S.W. (2000) 'Tabu search based heuristics for multi-floor facility layout', Int. J. Production Research, Vol. 38, No. 2, pp.365-383.

Castillo, I. and Peters, B.A. (2003) 'An extended distance-based facility layout problem', Int. J. Production Research, Vol. 41, No. 11, pp.2451-2479.

Cordeau J.-F., Gaudioso M, Laporte G, et al. A memetic heuristic for the generalized assignment problem. INFORMS J Comput. 2006; Vol. 18: pp. 433-443.

Cordeau J.-F., Gaudioso M, Laporte G, et al. The service allocation problem at the Gioia Tauro maritime terminal. European Journal of Operations Research. 2007; Vol. 176: pp. 1167-1184.

Dong, M., Wu, C. and Hou, F. (2009) 'Shortest path based simulated annealing algorithm for dynamic facility layout problem under dynamic business environment', Expert Systems with Applications, Vol. 36, No. 8, pp.11221-11232.

Francis, R. L., McGinnis, L.F., and White, J.A., 1992, "Facility Layout and Location: An Analytical Approach,” NJ: Prentice Hall.

Glover F. Future paths for integer programming and links to artificial intelligence. Comput Oper Res. 1986; Vol. 13: pp. 533-549.

Gunawan, A., Ng K.M., Poh, K.L. and Lau, C.H. (2014) 'Hybrid metaheuristics for solving the quadratic assignment problem and the generalized quadratic assignment problem', IEEE International Conference on Automation Science and Engineering (CASE).

Hahn PM, Kim B-J, Guignard M. An algorithm for the generalized quadratic assignment problem. Comput Optim Appl. 2008; Vol. 40: pp. 351-372.

Hamam, Y. and Hindi K.S. (2000) 'Assignment of program modules to processors: A simulated annealing approach', European Journal of Operational Research, Vol. 122, pp. 509-513.

Holland J.H. Adaptation in natural and artificial systems. Ann Arbor (MI): University of Michigan Press; 1975.

Lee C-G, Ma Z. The generalized quadratic assignment problem. Technical Report. Department of Mechanical and Industrial Engineering, University of Toronto, Toronto, ON; 2004. 
Liang, L.Y. and Chao, W.C. (2008) 'The strategies of tabu search technique for facility layout optimization', Automation in Construction, Vol. 17, pp.657-669.

Mateus G.R., Resende MGC, Silva RMA. GRASP with pathrelinking for the generalized quadratic assignment problem. J Heuristics. 2011; Vol. 17: pp. 527-565.

Matsuzaki, K., Irohara, T. and Yoshimoto, K. (1999) 'Heuristic algorithm to solve the multi-floor layout problem with the consideration of elevator utilization', Computers and Industrial Engineering, Vol. 36, pp.487-502.

McKendall A.R. (2008) "Improved Tabu search heuristics for the dynamic space allocation problem," Computers and Operations Research, Vol. 35, No.10, pp. 3347-59.

McKendall A.R., Jaramillo J.R. (2006) "A tabu search heuristic for the dynamic space allocation problem”. Computers and Operations Research, Vol. 32: pp. 107-125.

McKendall Jr., A.R., Shang, J. and Kuppusamy, S. (2006) 'Simulated annealing heuristics for the dynamic facility layout problem', Computers and Operations Research, Vol. 33, pp.2431-2444.

McKendall, A. and Li, C., 2017, “A tabu search heuristic for a generalized quadratic assignment problem,” Journal of Industrial and Production Engineering, Vol. 34, No. 3, pp. 221-231.

McKendall, A.R. and Hakobyan, A. (2010) 'Heuristics for the dynamic facility layout problem with unequal-area departments', European Journal of Operational Research, Vol. 201, No. 1, pp.171-182.

Metropolis N, Rosenbluth A, Rosenbluth M, Teller A, Teller M, 1953. Equation of state calculations by fast computing machines, Journal of Chemical Physics, Vol. 21, pp. 1087-1092.

Mir, M. and Imam, M.H. (2001) 'A hybrid optimization approach for layout design of unequalarea facilities', Computers and Industrial Engineering, Vol. 39, pp.49-63.

Pessoa AA, Hahn P.M., Guignard M, et al. Algorithms for the generalized quadratic assignment problem combining lagrangean decomposition and the reformulation linearization technique. Eur J Oper Res. 2010; Vol. 206: pp. 54-63.

Privault, C. and Herault, L. (1998) 'Solving a Real World Assignment Problem with a Metaheuristic', Journal of Heuristics, Vol. 4, pp. 383-398. 
Sahin, R., Ertogral, K. and Türkbey, O. (2010) 'A simulated annealing heuristic for the dynamic layout problem with budget constraint', Computers and Industrial Engineering, Vol. 59, pp.308313.

Samarghandi, H. and Eshghi, K. (2010) 'An efficient tabu algorithm for the single row facility layout problem', European Journal of Operational Research, Vol. 205, pp.98-105.

Scholz, D., Petrick, A. and Domschke, W. (2009) 'STaTS: a slicing tree and tabu search based heuristic for the unequal area facility layout problem', European Journal of Operational Research, Vol. 197, pp.166-178.

Seo, Y., Sheen, D., Moon, C. and Kim, T. (2006) 'Integrated design of workcells and unidirectional flowpath layout', Computers and Industrial Engineering, Vol. 51, pp.142-153.

Singh, S.P. (2009) 'Solving facility layout problem: three-level tabu search metaheuristic approach', Int. J. Recent Trends in Engineering, Vol. 1, No. 1, pp.73-77.

Souilah, A. (1995) 'Simulated annealing for manufacturing systems layout design', European Journal of Operational Research, Vol. 82, pp.592-614.

Sugiyono, A. (2006) 'Cellular manufacturing system application on redesign production layout with using heuristics algorithm', In 2006 IEEE International Conference on Management of Innovation and Technology, pp.940-944.

Tompkins, J.A., White, J.A., Bozer, Y.A., Frazelle, E.H., Tanchoco, J.M.A., and Trevino, J., 2003, "Facilities Planning," NY: John Wiley \& Sons, Inc.

Unal Y.Z., Uysal O. A new mixed integer programming model for curriculum balancing: application to a Turkish university. European Journal of Operations Research. 2014; Vol. 238: pp. 339-347.

Yang, T., Rajasekharan, M. and Peters, B.A. (1999) 'Semiconductor fabrication facility design using a hybrid search methodology', Computers and Industrial Engineering, Vol. 36, pp.565-583. 